2016

\title{
Global Strong Solutions of the Full Navier-Stokes and Q-Tensor System for Nematic Liquid Crystal Flows in Two Dimensions
}

\author{
Cecilia Cavaterra
}

Elisabetta Rocca

Hao Wu

Xiang Xu

Old Dominion University

Follow this and additional works at: https://digitalcommons.odu.edu/mathstat_fac_pubs

\section{Part of the Applied Mathematics Commons}

\section{Repository Citation}

Cavaterra, Cecilia; Rocca, Elisabetta; Wu, Hao; and Xu, Xiang, "Global Strong Solutions of the Full Navier-Stokes and Q-Tensor System for Nematic Liquid Crystal Flows in Two Dimensions" (2016). Mathematics \& Statistics Faculty Publications. 12.

https://digitalcommons.odu.edu/mathstat_fac_pubs/12

\section{Original Publication Citation}

Cavaterra, C., Rocca, E., Wu, H., \& Xu, X. (2016). Global strong solutions of the full Navier-Stokes and Q-tensor system for nematic liquid crystal flows in two dimensions. SIAM Journal on Mathematical Analysis, 48(2), 1368-1399. doi:10.1137/15M1048550 


\title{
GLOBAL STRONG SOLUTIONS OF THE FULL NAVIER-STOKES AND $Q$-TENSOR SYSTEM FOR NEMATIC LIQUID CRYSTAL FLOWS IN TWO DIMENSIONS*
}

\author{
CECILIA CAVATERRA ${ }^{\dagger}$, ELISABETTA ROCCA ${ }^{\ddagger}, \mathrm{HAO}_{\mathrm{WU}}^{\S}$, AND XIANG XU
}

\begin{abstract}
We consider a full Navier-Stokes and $Q$-tensor system for incompressible liquid crystal flows of nematic type. In the two dimensional periodic case, we prove the existence and uniqueness of global strong solutions that are uniformly bounded in time. This result is obtained without any smallness assumption on the physical parameter $\xi$ that measures the ratio between tumbling and aligning effects of a shear flow exerting over the liquid crystal directors. Moreover, we show the uniqueness of asymptotic limit for each global strong solution as time goes to infinity and provide an uniform estimate on the convergence rate.
\end{abstract}

Key words. nematic liquid crystal flow, $Q$-tensor system, global strong solution, uniqueness of asymptotic limit

AMS subject classifications. 35B44, 35D30, 35K45, 35Q30, 76A15

DOI. $10.1137 / 15 \mathrm{M} 1048550$

1. Introduction. In this paper, we study the global well-posedness and longtime dynamics of a full coupled incompressible Navier-Stokes and $Q$-tensor system due to Beris and Edwards [4], which models the evolution of incompressible liquid crystal flows of nematic type. In the Landau-de Gennes theory [12], the local orientation and degree of ordering for the liquid crystal molecules are characterized by a symmetric, traceless $d \times d$ tensor $Q$ (here $d$ stands for the spatial dimension), which measures the deviation of the second moment tensor from its isotropic value. The $Q$-tensor can incorporate the biaxiality of the liquid crystal molecule alignment [28]. Moreover, if $Q$ has two equal nonzero eigenvalues, then it can be formally written as $Q(x)=$ $s\left(n(x) \otimes n(x)-\frac{1}{d} \mathbb{I}\right)$ with $s \in \mathbb{R} \backslash\{0\}$ and the vector $n: \mathbb{R}^{d} \rightarrow \mathbb{S}^{d-1}$ representing the averaged macroscopic molecular orientation, so that the coupled $Q$-tensor system (see (1.1)-(1.3) below) reduces to the well-known Ericksen-Leslie system [24].

In this paper, we restrict ourselves to the periodic case. Denote by $\mathbb{T}^{d}$ the periodic box with period $a_{i}$ in the $i$ th direction and by $\mathcal{O}=\left(0, a_{1}\right) \times \cdots \times\left(0, a_{d}\right)$ the periodic cell. Without loss of generality, we can simply set $\mathcal{O}=(0,1)^{d}$. The coupled PDE sys-

*Received by the editors November 16, 2015; accepted for publication January 27, 2016; published electronically April 14, 2016. The research of the first and second authors was supported by FP7-IDEAS-ERC-StG 256872 (EntroPhase) and by GNAMPA (Gruppo Nazionale per l'Analisi Matematica, la Probabilità e le loro Applicazioni) of INdAM (Istituto Nazionale di Alta Matematica).

http://www.siam.org/journals/sima/48-2/M104855.html

${ }^{\dagger}$ Dipartimento di Matematica, Università degli Studi di Milano, 20133 Milano, Italy (cecilia. cavaterra@unimi.it).

${ }^{\ddagger}$ Weierstrass Institute for Applied Analysis and Stochastics, 10117 Berlin, Germany, and Dipartimento di Matematica, Università degli Studi di Milano, 20133 Milano, Italy (elisabetta. rocca@wias-berlin.de, elisabetta.rocca@unimi.it).

$\S$ Corresponding author. School of Mathematical Sciences and Shanghai Key Laboratory for Contemporary Applied Mathematics, Fudan University, 200433 Shanghai, People's Republic of China (haowufd@yahoo.com). The research of this author was partially supported by NNSFC under the grant 11371098 and Shanghai Center for Mathematical Sciences at Fudan University.

I Department of Mathematics and Statistics, Old Dominion University, Norfolk, VA 23529 (x2xu@odu.edu). The research of this author was supported by the start-up fund from the Department of Mathematics and Statistics at Old Dominion University.

1368 
tem we are going to study consists of incompressible Navier-Stokes equations for the fluid velocity with highly nonlinear anisotropic force terms and nonlinear convectiondiffusion equations of parabolic type that describe the evolution of the $Q$-tensor (see, e.g., [31]). More precisely, the full coupled Navier-Stokes and Q-tensor system takes the following form:

$$
\begin{aligned}
u_{t}+u \cdot \nabla u-\nu \Delta u+\nabla P & =\lambda \nabla \cdot(\tau+\sigma), & & (x, t) \in \mathbb{T}^{d} \times \mathbb{R}^{+}, \\
\nabla \cdot u & =0, & & (x, t) \in \mathbb{T}^{d} \times \mathbb{R}^{+}, \\
Q_{t}+u \cdot \nabla Q-S(\nabla u, Q) & =\Gamma H(Q), & & (x, t) \in \mathbb{T}^{d} \times \mathbb{R}^{+} .
\end{aligned}
$$

Here, the vector $u(x, t): \mathbb{T}^{d} \times(0,+\infty) \rightarrow \mathbb{R}^{d}$ denotes the velocity field of the fluid and $Q(x, t): \mathbb{T}^{d} \times(0,+\infty) \rightarrow S_{0}^{(d)}$ stands for the order parameter of liquid crystal molecules. (See (2.1) for the definition of the set $S_{0}^{(d)}$.) We assume that the system (1.1)-(1.3) is subject to the periodic boundary conditions

$$
u\left(x+e_{i}, t\right)=u(x, t), \quad Q\left(x+e_{i}, t\right)=Q(x, t) \text { for }(x, t) \in \mathbb{T}^{d} \times \mathbb{R}^{+},
$$

where $\left\{e_{i}\right\}_{i=1}^{d}$ is the canonical orthonormal basis of $\mathbb{R}^{d}$. Moreover, the system is subject to the spatially 1-periodic initial data

$$
\left.u\right|_{t=0}=u_{0}(x) \text { with } \nabla \cdot u_{0}=0,\left.\quad Q\right|_{t=0}=Q_{0}(x) \quad \text { for } x \in \mathbb{T}^{d} .
$$

We note that the system preserves for all time both the symmetry and tracelessness of any solution $Q$ associated to an initial datum with the same property [31,40].

The system (1.1)-(1.3) describes the complex interaction between the fluid and the alignment of liquid crystal molecules: the evolution of the fluid affects the direction and position of the molecules, while changes in the alignment of molecules will also influence the fluid velocity. The positive constants $\nu, \lambda$, and $\Gamma$ stand for the fluid viscosity, the competition between kinetic energy and elastic potential energy, and the macroscopic elastic relaxation time (Deborah number) for the molecular orientation field, respectively.

The free energy for liquid crystal molecules is given by (see, e.g., [28])

$$
\mathcal{F}(Q) \stackrel{\text { def }}{=} \int_{\mathbb{T}^{d}}\left(\frac{L}{2}|\nabla Q|^{2}+f_{B}(Q)\right) d x .
$$

In the definition of $\mathcal{F}(Q)$, the gradient term corresponds to the elastic part of the free energy and $L>0$ is the elastic constant. Here, we simply use the so-called one constant approximation of the general Oseen-Frank energy (cf. [3]). On the other hand, the bulk part $f_{B}(Q)$ of Landau-de Gennes type takes the form

$$
f_{B}(Q)=\frac{a}{2} \operatorname{tr}\left(Q^{2}\right)-\frac{b}{3} \operatorname{tr}\left(Q^{3}\right)+\frac{c}{4} \operatorname{tr}^{2}\left(Q^{2}\right),
$$

where $a, b, c \in \mathbb{R}$ are material-dependent and temperature-dependent coefficients that are assumed to be constants here for simplicity. In particular, throughout the paper we assume that

$$
c>0,
$$

which is necessary from the modeling point of view to guarantee that the free energy $\mathcal{F}(Q)$ (and also the total energy $\mathcal{E}(t)$ of the coupled system (1.1)-(1.3), see (2.3)) is bounded from below (see $[27,28])$. 
The tensor $H=H(Q)$ in (1.3) is related to the variational derivative of the free energy $\mathcal{F}(Q)$ with respect to $Q$ (under the constraint that $Q$ is both symmetric and traceless) such that

$$
H(Q) \stackrel{\text { def }}{=}-\frac{\partial \mathcal{F}(Q)}{\partial Q}=L \Delta Q-a Q+b\left(Q^{2}-\frac{1}{d} \operatorname{tr}\left(Q^{2}\right) \mathbb{I}\right)-c Q \operatorname{tr}\left(Q^{2}\right),
$$

where $\mathbb{I} \in \mathbb{R}^{d \times d}$ stands for the identity matrix. Besides, the matrix valued function $S(\nabla u, Q)$ in (1.3) takes the form

$$
\begin{aligned}
S(\nabla u, Q) \stackrel{\text { def }}{=} & (\xi D+\Omega)\left(Q+\frac{1}{d} \mathbb{I}\right)+\left(Q+\frac{1}{d} \mathbb{I}\right)(\xi D-\Omega) \\
& -2 \xi\left(Q+\frac{1}{d} \mathbb{I}\right) \operatorname{tr}(Q \nabla u),
\end{aligned}
$$

where

$$
D=\frac{\nabla u+\nabla^{T} u}{2}, \quad \Omega=\frac{\nabla u-\nabla^{T} u}{2}
$$

represent the symmetric and skew-symmetric parts of the rate of strain tensor, respectively. We note that $S(\nabla u, Q)$ describes the rotating and stretching effects on the order parameter $Q$ due to the fluid, as the liquid crystal molecules can be tumbled and aligned by the flow. In particular, the constant parameter $\xi \in \mathbb{R}$ in (1.8) depends on the molecular shapes of the liquid crystal, and it is a measure of the ratio between the tumbling and the aligning effect that a shear flow exerts on the liquid crystal directors.

Concerning the stress tensors $\tau$ and $\sigma$ on the right-hand side of Navier-Stokes equations (1.1), the symmetric part $\tau$ reads

$$
\begin{aligned}
\tau \stackrel{\text { def }}{=} & -\xi\left(Q+\frac{1}{d} \mathbb{I}\right) H(Q)-\xi H(Q)\left(Q+\frac{1}{d} \mathbb{I}\right)+2 \xi\left(Q+\frac{1}{d} \mathbb{I}\right) \operatorname{tr}(Q H(Q)) \\
& -L \nabla Q \odot \nabla Q,
\end{aligned}
$$

in which the last term is understood as $(\nabla Q \odot \nabla Q)_{i j}=\sum_{k, l=1}^{d} \nabla_{i} Q_{k l} \nabla_{j} Q_{k l}$. On the other hand, the skew-symmetric part $\sigma$ is given by

$$
\sigma \stackrel{\text { def }}{=} Q H(Q)-H(Q) Q .
$$

We recall some related results in the literature. The coupled Beris-Edwards system (1.1)-(1.3) has been recently studied by several authors. For the simpler case with $\xi=0$, which means that the molecules only tumble in a shear flow but are not aligned by the flow (cf. [32]), the first contribution is due to [32], in which the authors proved the existence of global weak solutions to the Cauchy problem in $\mathbb{R}^{d}$ with $d=2,3$, and they obtained higher global regularity as well as the weak-strong uniqueness for $d=2$. Asymptotic behavior of the Cauchy problem in $\mathbb{R}^{3}$ with $\xi=0$ is recently discussed in [10]. Besides, initial boundary value problems subject to various boundary conditions for $d=2,3$ have been investigated by several authors in $[2,16,17]$ under the assumption $\xi=0$. In these works, they proved the existence of global weak solutions, the existence and uniqueness of local strong solutions, as well as some regularity criteria, etc. For the full Navier-Stokes and $Q$-tensor system (1.1)-(1.3) with general $\xi \in \mathbb{R}$, the existence of global weak solutions for the Cauchy problem in 
$\mathbb{R}^{d}$ with $d=2,3$ was established in [31] for sufficiently small $|\xi|$, while the uniqueness of weak solutions in the two dimensional (2D) setting is given quite recently in [11]. On the other hand, in [1] the authors proved the existence of global weak solutions and local well-posedness with higher time-regularity for the initial boundary value problem subject to inhomogeneous mixed Dirichlet/Neumann boundary conditions.

Some recent progress has also been made on the mathematical analysis of certain modified versions of the Beris-Edwards system in terms of its free energy. For instance, in [40], the regular bulk potential in (1.6) is replaced by a singular potential of Ball-Majumdar type (cf. [3]) that ensures the $Q$-tensor always stays in the "physical" region. Then, in the co-rotational regime $\xi=0$, the author proved the existence of global weak solutions for $d=2,3$, and for $d=2$ he further obtained the existence and uniqueness of global regular solutions. In $[13,14]$, nonisothermal variants of the Beris-Edwards system were derived and the authors proved the existence of global weak solutions in the case of a singular potential under periodic boundary conditions for general $\xi \in \mathbb{R}$ and $d=3$. In [19], the authors considered a general Beris-Edwards system where the Dirichlet type elastic functional as in (1.6) is replaced by three quadratic functionals. For the Cauchy problem in $\mathbb{R}^{3}$, they proved the existence of global weak solutions as well as the existence of a unique global strong solution provided that the fluid viscosity is sufficiently large. We also refer interested readers to $[9,22]$ for well-posedness results regarding the $Q$-tensor gradient flow generated by the general Landau-de Gennes energy with a cubic term (but without fluid coupling).

It is worth mentioning that a rigorous derivation from the Beris-Edwards system (with general free energy and arbitrary $\xi$ ) to the classical Ericksen-Leslie system is recently given in [39] by using the Hilbert expansion method. We refer to $[6,7,20,26$, $38,42]$ and the references therein for mathematical analysis of the general EricksenLeslie system either under the unit length constraint of the molecule director or with Ginzburg-Landau approximation of the free energy.

In this paper, we are interested in the global well-posedness and long-time behavior of the full Navier-Stokes and $Q$-tensor system (1.1)-(1.5) in the 2D periodic setting. The main difficulty in handling the current full coupled system with $\xi \in \mathbb{R}$ is due to the fact that for $\xi \neq 0$ the system (1.1)-(1.5) no longer enjoys certain maximum principle for the $Q$-equation (1.3), which is instead true in the case in which $\xi=0$ (see, e.g., [17, Theorem 3]). Due to the loss of control on $Q$ in $L^{\infty}\left(0, T ; L^{\infty}\right)$, extra difficulties arise in obtaining estimates for those highly nonlinear terms of the system (see Proposition 12). We note that a similar problem was encountered in [31] in proving the existence of global strong solutions of the Cauchy problem in $\mathbb{R}^{2}$ (assuming that $|\xi|$ is sufficiently small). In order to get such high nonlinearities under control, the authors of [31] chose to work within the technical Littlewood-Paley approach and then made use of the sharp logarithmic Sobolev embedding of $H^{1+\epsilon}$ in $L^{\infty}$ (cf. [5]) together with the precise growth of the constant for the Sobolev embedding of $H^{1}$ in $L^{p}$ for any $p>1$ (cf. [8]), and an optimal choice of the nonconstant index $p$ of interpolation depending on the norm of the solution. Then they established the existence of a unique global strong solution $(u, Q)$ to the Cauchy problem in $\mathbb{R}^{2}$, whose $H^{s} \times H^{1+s}$-norm $(s>0)$ may increase at most quadruply exponential in time.

We note that in [31], smallness of the parameter $|\xi|$ is required because of the unboundedness of the whole plane $\mathbb{R}^{2}$, which, however, can be removed in our current periodic setting (see (2.8)). In the periodic domain $\mathbb{T}^{2}$, the first main result we are able to prove is the existence and uniqueness of global strong solutions $(u, Q)$ for arbitrary $\xi \in \mathbb{R}$, whose $H^{1} \times H^{2}$-norm is indeed uniformly bounded in time (see Theorem 6). To achieve this goal, we use the idea of [25] for the simplified liquid crystal system together 
with the interpolation techniques in [31] to derive a suitable higher-order differential inequality for a specific quantity $\mathcal{A}(t)$ (see (3.5) for its definition), which is essentially contained in the energy dissipation of the system (1.1)-(1.5) and is integrable with respect to time on the unbounded half line $\mathbb{R}^{+}$such that $\mathcal{A}(t) \in L^{1}(0,+\infty)$ (see Proposition 12). The resulting higher-order energy inequality (3.6) has a delicate double-logarithmic type structure, and it plays a crucial role in three aspects of the subsequent proofs: (1) it yields uniform-in-time estimates on $H^{1} \times H^{2}$-norm of the global strong solution $(u, Q)$ provided that $\left(u_{0}, Q_{0}\right) \in H^{1} \times H^{2}$ (see (4.19)); (2) it implies the decay of $\mathcal{A}(t)$ to zero as $t \rightarrow+\infty$ and thus gives a characterization of the $\omega$-limit set of the evolution system (1.1)-(1.5) (see Lemma 18); and (3) it helps to obtain an uniform estimate on the rate of convergence to equilibrium for the global strong solution (see (5.38)).

Our second main result is about the long-time behavior of global strong solutions obtained in Theorem 6 (see Theorem 7). The problem of whether a bounded global solution of a nonlinear evolution equation will converge to a single equilibrium as time tends to infinity is of great importance. This issue is nontrivial since the structure of the equilibrium set may form a continuum for many dynamical systems in higher spatial dimensions. For instance, under the current periodic boundary conditions, it is expected that the dimension of the equilibrium set for our problem (1.1)-(1.5) is at least 2 due to the simple fact that a shift in each variable produces another steady state. Hence, it is interesting to determine whether a trajectory defined by a global strong solution will converge to a single steady state or not. To this end, we first construct a specific gradient inequality for tensor valued functions subject to periodic boundary conditions (see Lemma 19), and then we apply the LojasiewiczSimon approach (see [34] and also [15]) to achieve our aim. This approach turns out to be a powerful tool in the study of long-time dynamics of evolution equations, and we refer interested readers to [21] and the references therein for various applications.

The rest of this paper is organized as follows. In section 2 we introduce the notation as well as some preliminary results and then state the main results of this paper. Section 3 is devoted to the derivation of a specific higher-order differential inequality that will be crucial in the subsequent proof. In section 4, we prove the existence and uniqueness of global strong solutions to the Beris-Edward system (1.1)(1.5). In section 5 we demonstrate that every global strong solution will converge to a single equilibrium and provide a uniform estimate on the convergence rate. Some detailed calculations will be presented in the appendix.

\section{Preliminaries and main results.}

2.1. Notation. Let $X$ be a real Banach space with norm $\|\cdot\|_{X}$ and $X^{*}$ be its dual space. By $\langle\cdot, \cdot\rangle_{X^{*}, X}$ we indicate the duality product between $X$ and $X^{*}$. We denote by $L^{p}\left(\mathbb{T}^{d}, M\right), W^{m, p}\left(\mathbb{T}^{d}, M\right)$ the usual Lebesgue and Sobolev spaces defined on the torus $\mathbb{T}^{d}$ for $M$-valued functions (e.g., $M=\mathbb{R}, M=\mathbb{R}^{d}$ or $M=\mathbb{R}^{d} \times \mathbb{R}^{d}$ ) that are in $L_{l o c}^{p}\left(\mathbb{R}^{d}\right)$ or $W_{l o c}^{m, p}\left(\mathbb{R}^{d}\right)$ and periodic in $\mathbb{T}^{d}$, with norms denoted by $\|\cdot\|_{L^{p}}$, $\|\cdot\|_{W^{m, p}}$, respectively. For $p=2$, we simply denote $H^{m}\left(\mathbb{T}^{d}\right)=W^{m, 2}\left(\mathbb{T}^{d}\right)$ with norm $\|\cdot\|_{H^{m}}$. In particular for $m=0$, we denote $H^{0}\left(\mathbb{T}^{d}\right)=L^{2}\left(\mathbb{T}^{d}\right)$ and the inner product on $L^{2}\left(\mathbb{T}^{d}\right)$ will be denoted by $(\cdot, \cdot)_{L^{2}}$. For simplicity, we shall not distinguish functional spaces when scalar-valued, vector-valued, or matrix-valued functions are involved if they are clear from the context.

The Einstein summation convention will be used throughout this paper. For arbitrary vectors $u, v \in \mathbb{R}^{d}$, we denote $u \cdot v=u_{i} v_{i}$ the inner product in $\mathbb{R}^{d}$. For any matrix $Q \in \mathbb{R}^{d \times d}$, the Frobenius norm $|Q|=\sqrt{\operatorname{tr}\left(Q^{2}\right)}=\sqrt{Q_{i j} Q_{i j}}$ will be used. Let 
$S_{0}^{(d)}$ denote the space of symmetric traceless matrices with spatial dimension $d$,

$$
S_{0}^{(d)} \stackrel{\text { def }}{=}\left\{Q \in \mathbb{R}^{d \times d} \mid Q_{i j}=Q_{j i}, \operatorname{tr}(Q)=0, i, j=1, \ldots, d\right\} .
$$

Then for matrices $A, B \in S_{0}^{(d)}$, we denote $A: B=\operatorname{tr}(A B)$. Concerning the norms for spatial derivatives of matrices, we denote $|\nabla Q|^{2}(x)=\nabla_{k} Q_{i j}(x) \nabla_{k} Q_{i j}(x)$ and $|\Delta Q|^{2}(x)=\Delta Q_{i j}(x) \Delta Q_{i j}(x)$. Then Sobolev spaces for $Q$-tensors will be defined in terms of the above norms. For instance,

$$
\begin{aligned}
L^{2}\left(\mathbb{T}^{d}, S_{0}^{(d)}\right) & =\left\{Q: \mathbb{T}^{d} \rightarrow S_{0}^{(d)}, \int_{\mathbb{T}^{d}}|Q(x)|^{2} d x<\infty\right\}, \\
H^{1}\left(\mathbb{T}^{d}, S_{0}^{(d)}\right) & =\left\{Q: \mathbb{T}^{d} \rightarrow S_{0}^{(d)}, \int_{\mathbb{T}^{d}}|\nabla Q(x)|^{2}+|Q(x)|^{2} d x<\infty\right\} .
\end{aligned}
$$

Concerning the divergence of a $d \times d$ differentiable matrix-valued function $\sigma=\left(\sigma_{i j}\right)$, its $i$ th component is given by $(\nabla \cdot \sigma)_{i}=\nabla_{j} \sigma_{i j}, 1 \leq i, j \leq d$.

For any normed space $X$, the subspace of functions in $X$ with zero-mean will be denoted by $\dot{X}$, that is, $\dot{X}=\left\{v \in X: \int_{\mathbb{T} d} v d x=0\right\}$. Then we recall the wellestablished functional settings for periodic solutions to Navier-Stokes equations (see, e.g., $[36])$ :

$$
\begin{aligned}
\mathbf{H} & =\left\{v \in \dot{L}^{2}\left(\mathbb{T}^{d}, \mathbb{R}^{d}\right), \nabla \cdot v=0\right\}, \\
\mathbf{V} & =\left\{v \in \dot{H}^{1}\left(\mathbb{T}^{d}, \mathbb{R}^{d}\right), \nabla \cdot v=0\right\}, \\
\mathbf{V}^{\prime} & =\text { the dual space of } \mathbf{V} .
\end{aligned}
$$

In the spatial periodic setting, one can define a mapping $\mathcal{S}$ associated with the Stokes problem:

$$
\mathcal{S} u=-\Delta u \quad \forall u \in D(\mathcal{S}) \stackrel{\text { def }}{=}\{u \in \mathbf{H}, \Delta u \in \mathbf{H}\}=\dot{H}^{2}\left(\mathbb{T}^{d}, \mathbb{R}^{d}\right) \cap \mathbf{H} .
$$

The operator $\mathcal{S}$ can be seen as an unbounded positive linear self-adjoint operator on $\mathbf{H}$. If $D(\mathcal{S})$ is endowed with the norm induced by $\dot{H}^{2}\left(\mathbb{T}^{d}\right)$, then $\mathcal{S}$ becomes an isomorphism from $D(\mathcal{S})$ onto $\mathbf{H}$. For detailed properties of $\mathcal{S}$, we refer readers to [36].

We denote by $C$ a generic constant that may depend on $\nu, \Gamma, \lambda, \xi, L, a, b, c, \mathbb{T}^{d}$ and the initial data $\left(u_{0}, Q_{0}\right)$, whose value is allowed to vary on occurrence. Specific dependence will be pointed out explicitly if necessary.

2.2. Basic energy law and global weak solutions. We first present some basic properties of the Navier-Stokes and $Q$-tensor system (1.1)-(1.5) that are valid in both $2 \mathrm{D}$ and three dimensional (3D) cases.

The total energy of the system (1.1)-(1.5) consists of two parts: the kinetic energy for the velocity field $u$ and the free energy $\mathcal{F}(Q)$ (see (1.6)). More precisely, we have

$$
\mathcal{E}(t) \stackrel{\text { def }}{=} \frac{1}{2} \int_{\mathbb{T}^{d}}|u|^{2}(x, t) d x+\lambda \mathcal{F}(Q(t)) .
$$

By the same argument as in [31, Proposition 1] for the whole space case in $\mathbb{R}^{d}$ (see also [11, Proposition 2.1]), we can derive the following basic energy law.

Lemma 1 (basic energy law). Suppose $d=2,3$. Let $(u, Q)$ be a smooth solution to the problem (1.1)-(1.5). Then we have

$$
\frac{d}{d t} \mathcal{E}(t)=-\nu \int_{\mathbb{T}^{d}}|\nabla u|^{2} d x-\lambda \Gamma \int_{\mathbb{T}^{d}}|H(Q)|^{2} d x \leq 0 \quad \forall t>0 .
$$

Copyright $@$ by SIAM. Unauthorized reproduction of this article is prohibited. 
Lemma 1 reflects the energy dissipation of the liquid crystal flow and indicates that the energy functional $\mathcal{E}(t)$, which is bounded from below since $c>0$, serves as a Lyapunov functional for the system (1.1)-(1.5). This property provides necessary global estimates for further mathematical analysis of the PDE system (1.1)-(1.5), for instance, the existence of global weak solutions.

Lemma 2. Suppose $d=2,3$. Let $(u, Q)$ be a smooth solution to the problem (1.1)-(1.5). Then we have

$$
\|u(t)\|_{L^{2}}+\|Q(t)\|_{H^{1}} \leq C \quad \forall t>0,
$$

where the constant $C>0$ depends on $\left\|u_{0}\right\|,\left\|Q_{0}\right\|_{H^{1}}, L, \lambda, a, b, c$ and $\mathbb{T}^{d}$. Moreover, it holds that

$$
\int_{0}^{T} \int_{\mathbb{T}^{d}}|\nabla u(x, t)|^{2}+|\Delta Q(x, t)|^{2} d x d t \leq C_{T} \quad \forall T>0,
$$

where $C_{T}>0$ may further depend on $\nu, \Gamma$, and $T$.

Proof. It follows from Lemma 1 that

$$
\mathcal{E}(t)+\int_{0}^{t} \int_{\mathbb{T}^{d}} \nu|\nabla u|^{2}+\lambda \Gamma|H(Q)|^{2} d x d t=\mathcal{E}(0) \quad \forall t>0 .
$$

We easily infer from the Sobolev embedding theorem $(d=2,3)$ that

$$
\mathcal{E}(0)=\frac{1}{2}\left\|u_{0}\right\|_{L^{2}}^{2}+\lambda \mathcal{F}\left(Q_{0}\right) \leq C\left(\left\|u_{0}\right\|_{L^{2}},\left\|Q_{0}\right\|_{H^{1}}\right) .
$$

On the other hand, there exists a constant $M=M(a, b, c)>0$ large enough (see [31, (18)]) such that

$$
\frac{M}{2} \operatorname{tr}\left(Q^{2}\right)+\frac{c}{8} \operatorname{tr}^{2}\left(Q^{2}\right) \leq\left(M+\frac{a}{2}\right) \operatorname{tr}\left(Q^{2}\right)-\frac{b}{3} \operatorname{tr}\left(Q^{3}\right)+\frac{c}{4} \operatorname{tr}^{2}\left(Q^{2}\right),
$$

which combined with the Young's inequality and the fact $c>0$ yields that

$$
\begin{aligned}
\frac{a}{2} \operatorname{tr}\left(Q^{2}\right)-\frac{b}{3} \operatorname{tr}\left(Q^{3}\right)+\frac{c}{4} \operatorname{tr}^{2}\left(Q^{2}\right) & \geq-\frac{M}{2} \operatorname{tr}\left(Q^{2}\right)+\frac{c}{8} \operatorname{tr}^{2}\left(Q^{2}\right) \\
& \geq \frac{1}{2} \operatorname{tr}\left(Q^{2}\right)+\frac{c}{16} \operatorname{tr}^{2}\left(Q^{2}\right)-\frac{(M+1)^{2}}{c} .
\end{aligned}
$$

Then we have the estimate

$$
\begin{aligned}
& \frac{1}{2}\|u(t)\|_{L^{2}}^{2}+\frac{\lambda L}{2}\|\nabla Q(t)\|_{L^{2}}^{2}+\lambda \int_{\mathbb{T}^{d}} \frac{1}{2} \operatorname{tr}\left(Q^{2}(t)\right)+\frac{c}{16} \operatorname{tr}^{2}\left(Q^{2}(t)\right) d x \\
& \quad \leq \frac{1}{2}\|u(t)\|^{2}+\lambda \mathcal{F}(Q(t))+\frac{\lambda(M+1)^{2}}{c}\left|\mathbb{T}^{d}\right| \quad \forall t>0,
\end{aligned}
$$

where $\left|\mathbb{T}^{d}\right|$ stands for the Lebesgue measure of $\mathbb{T}^{d}$. As a consequence, we can deduce that $\mathcal{E}(t)$ is uniformly bounded from below by a generic constant only depending on the coefficients $a, b, c, \lambda$ and the size of periodic domain. Hence, the estimate (2.5) easily follows from (2.7) and (2.8).

Copyright $@$ by SIAM. Unauthorized reproduction of this article is prohibited. 
Next, we infer from (2.5), (2.7), (2.8), and the Sobolev embedding theorem $(d=$ $2,3)$ that

$$
\begin{aligned}
\int_{0}^{t} & \int_{\mathbb{T}^{d}}|L \Delta Q(\tau)|^{2} d x d \tau \\
\leq & 2 \int_{0}^{t} \int_{\mathbb{T}^{d}}|H(Q(\tau))|^{2} d x d \tau \\
& +2 \int_{0}^{t} \int_{\mathbb{T}^{d}}\left|-a Q+b\left(Q^{2}-\frac{1}{3} \operatorname{tr}\left(Q^{2}\right) \mathbb{I}\right)-c Q \operatorname{tr}\left(Q^{2}\right)\right|^{2} d x d \tau \\
& \leq 2 \int_{0}^{t} \int_{\mathbb{T}^{d}}|H(Q)|^{2} d x d \tau+C \int_{0}^{t}\left(\|Q(\tau)\|_{L^{2}}^{2}+\|Q(\tau)\|_{L^{4}}^{4}+\|Q(\tau)\|_{L^{6}}^{6}\right) d \tau \\
& \leq \frac{2}{\lambda \Gamma}(\mathcal{E}(0)-\mathcal{E}(t))+C t \\
& \leq C(1+t) \quad \forall t>0,
\end{aligned}
$$

where $C$ depends on $\left\|u_{0}\right\|,\left\|Q_{0}\right\|_{H^{1}}, \Gamma, L, \lambda, a, b, c$, and $\mathbb{T}^{d}$. Then the conclusion (2.6) easily follows from the above estimate and (2.7).

Remark 3. For the full Navier-Stokes and Q-tensor system (1.1)-(1.5) with general parameter $\xi \in \mathbb{R}$, the existence of global weak solutions for the Cauchy problem in the whole space $\mathbb{R}^{d}$ with $d=2,3$ is established in [31] for sufficiently small $\xi$. On the other hand, for the initial boundary value problem in a bounded domain in $\mathbb{R}^{d}$, in [1] the existence of global weak solutions under inhomogeneous mixed Dirichlet/Neumann boundary conditions was obtained without any restriction on $\xi$. The smallness for $\xi$ can be removed in the bounded domain case because one can use a generic constant depending on the domain size to get a priori $L^{2}$-estimate for the $Q$-tensor (see (2.8)).

Since we are working with the periodic domain, the following result can be easily proved in a way similar to [1].

Proposition 4 (existence of global weak solutions). Suppose that $d=2,3$ and $\xi \in \mathbb{R}$. For any initial data $\left(u_{0}, Q_{0}\right) \in \mathbf{H} \times H^{1}\left(\mathbb{T}^{d}, S_{0}^{(d)}\right)$ and $T>0$, the problem (1.1)-(1.5) possesses at least one global weak solution $(u, Q)$ such that

$$
\begin{aligned}
& u \in L^{\infty}(0, T ; \mathbf{H}) \cap L^{2}(0, T ; \mathbf{V}), \\
& Q \in L^{\infty}\left(0, T ; H^{1}\left(\mathbb{T}^{d}, S_{0}^{(d)}\right)\right) \cap L^{2}\left(0, T ; H^{2}\left(\mathbb{T}^{d}, S_{0}^{(d)}\right)\right) .
\end{aligned}
$$

Moreover, for a.e. $t \in(0, T)$, the following energy inequality holds:

$$
\mathcal{E}(t)+\int_{0}^{t} \int_{\mathbb{T}^{d}} \nu|\nabla u|^{2}+\lambda \Gamma|H(Q)|^{2} d x d t \leq \mathcal{E}(0) .
$$

2.3. Main results. In the remainder of this paper, we shall focus on the $2 \mathrm{D}$ case that $d=2$. First, we observe the simple fact that

$$
\operatorname{tr}\left(Q^{3}\right)=0 \quad \text { when } d=2,
$$

and thus the cubic term with coefficient $b$ in the free energy $\mathcal{F}(Q)$ (see (1.6)) just vanishes (cf. [22]). As a consequence, we have a simpler expression for $H(Q)$ in the $2 \mathrm{D}$ case:

$$
H(Q)=L \Delta Q-a Q-c Q \operatorname{tr}\left(Q^{2}\right)
$$

Let us introduce the notion of strong solutions to the problem (1.1)-(1.5).

Copyright $@$ by SIAM. Unauthorized reproduction of this article is prohibited. 
Definition 5. Suppose that $d=2$ and $\left(u_{0}, Q_{0}\right) \in \mathbf{V} \times H^{2}\left(\mathbb{T}^{2}, S_{0}^{(2)}\right) . \quad A$ pair $(u, Q)$ is called a global strong solution to the problem (1.1)-(1.5) if

$$
\begin{aligned}
& u \in C([0,+\infty) ; \mathbf{V}) \cap L_{l o c}^{2}\left(0,+\infty ; H^{2}\left(\mathbb{T}^{2}, \mathbb{R}^{2}\right)\right), \\
& Q \in C\left([0,+\infty) ; H^{2}\left(\mathbb{T}^{2}, S_{0}^{(2)}\right)\right) \cap L_{l o c}^{2}\left(0,+\infty ; H^{3}\left(\mathbb{T}^{2}, S_{0}^{(2)}\right)\right) .
\end{aligned}
$$

Moreover, (1.1) for $u$ and (1.3) for $Q$ are satisfied in $L_{l o c}^{2}(0,+\infty ; \mathbf{H})$ and $L_{\text {loc }}^{2}\left(0,+\infty ; L^{2}\left(\mathbb{T}^{2}, S_{0}^{(2)}\right)\right)$, respectively.

Then we state the main results of this paper. The first result is about the global well-posedness of the hydrodynamic system (1.1)-(1.5) in $\mathbb{T}^{2}$.

TheOREm 6 (existence and uniqueness of global strong solutions). Suppose $d=$ 2 and $\xi \in \mathbb{R}$. Then for any $\left(u_{0}, Q_{0}\right) \in \mathbf{V} \times H^{2}\left(\mathbb{T}^{2}, S_{0}^{(2)}\right)$, the problem (1.1)-(1.5) admits a unique global strong solution $(u, Q)$ in the sense of Definition 5 , which satisfies

$$
\|u(t)\|_{H^{1}}+\|Q(t)\|_{H^{2}} \leq C \quad \forall t \geq 0
$$

where $C>0$ is a constant that depends on $\nu, \Gamma, L, \lambda, a, c, \mathbb{T}^{2},\left\|u_{0}\right\|_{H^{1}},\left\|Q_{0}\right\|_{H^{2}}$, and $\xi$ but not on $t$.

Our second main result states that for any global strong solution obtained in Theorem 6 , it has an unique asymptotic limit as $t \rightarrow+\infty$.

TheOrem 7 (uniqueness of asymptotic limit). Suppose that the assumptions in Theorem 6 are satisfied. For any $\left(u_{0}, Q_{0}\right) \in \mathbf{V} \times H^{2}\left(\mathbb{T}^{2}, S_{0}^{(2)}\right)$, the unique global strong solution to the problem (1.1)-(1.5) converges to a single steady state solution $\left(0, Q_{\infty}\right)$ as time tends to infinity:

$$
\lim _{t \rightarrow+\infty}\left(\|u(t)\|_{H^{1}}+\left\|Q(t)-Q_{\infty}\right\|_{H^{2}}\right)=0,
$$

where $Q_{\infty} \in S_{0}^{(2)}$ satisfies the elliptic problem in $\mathbb{T}^{2}$

$$
L \Delta Q_{\infty}-a Q_{\infty}-c \operatorname{tr}\left(Q_{\infty}^{2}\right) Q_{\infty}=0, \text { in } \mathbb{T}^{2}, \quad Q_{\infty}\left(x+e_{i}\right)=Q_{\infty} \text { for } x \in \mathbb{T}^{2} .
$$

Furthermore, the following estimate on convergence rate holds:

$$
\|u(t)\|_{H^{1}}+\left\|Q(t)-Q_{\infty}\right\|_{H^{2}} \leq C(1+t)^{-\frac{\theta}{1-2 \theta}} \quad \forall t \geq 0 .
$$

Here, $C>0$ is a constant that depends on $\nu, \Gamma, L, \lambda, a, c, \xi, \mathbb{T}^{2},\left\|u_{0}\right\|_{H^{1}},\left\|Q_{0}\right\|_{H^{2}}$, and $\left\|Q_{\infty}\right\|_{H^{2}}$, and $\theta \in\left(0, \frac{1}{2}\right)$ is the constant given in Lemma 19 depending on $Q_{\infty}$.

3. Higher-order energy inequality. In this section we will derive a useful higher-order energy inequality for the problem (1.1)-(1.5). For the sake of simplicity, the subsequent calculations shall be performed formally on smooth solutions of the problem (1.1)-(1.5), without referring to any approximation. Nevertheless, they can be justified by working within the Faedo-Galerkin approximation scheme (4.1)-(4.7) described in section 4 .

We start by recalling the following special cases of the Gagliardo-Nirenberg inequality in 2D that will be frequently used in the subsequent proofs (see, e.g., [23]).

Lemma 8. Suppose $d=2$. We have

$$
\begin{aligned}
\|g\|_{L^{4}} & \leq C\left(\|\nabla g\|_{L^{2}}^{\frac{1}{2}}\|g\|_{L^{2}}^{\frac{1}{2}}+\|g\|_{L^{2}}\right) \quad \forall g \in H^{1}\left(\mathbb{T}^{2}\right), \\
\|\nabla g\|_{L^{2}} & \leq\|g\|_{L^{2}}^{\frac{1}{2}}\|\Delta g\|_{L^{2}}^{\frac{1}{2}} \quad \forall g \in H^{2}\left(\mathbb{T}^{2}\right) .
\end{aligned}
$$

Copyright $@$ by SIAM. Unauthorized reproduction of this article is prohibited. 
Besides, we will make use of the following $L^{p}$-interpolation inequality with precise growth of the constant in 2D, which follows from [8] (see also [30, Lemma 10]) and the Sobolev extension theorem [29, Chapter 2, section 3.6].

Lemma 9. Suppose $d=2$. For any $\eta>1$, it holds that

$$
\|g\|_{L^{2 \eta}} \leq C \sqrt{\eta}\|g\|_{H^{1}}^{1-\frac{1}{\eta}}\|g\|_{L^{2}}^{\frac{1}{\eta}} \quad \forall g \in H^{1}\left(\mathbb{T}^{2}\right),
$$

where the constant $C$ is independent of the exponent $\eta$ and the function $g$.

Next, we recall that when $\xi=0$ the system (1.1)-(1.5) enjoys a maximum principle for the $Q$-equation (1.3) (see, e.g., [17, Theorem 3]). However, since now the parameter $\xi$ is allowed to be nonzero, the maximum principle property is no longer valid. The loss of control on the $L_{t}^{\infty} L_{x}^{\infty}$-norm of $Q$ brings us several difficulties in obtaining estimates for highly nonlinear terms of the system. In order to handle the $L^{\infty}$-norm of $Q$, we shall use the following well-known results.

Lemma 10 (Agmon's inequality [37]). When $d=2$, it holds that

$$
\|g\|_{L^{\infty}} \leq C\|g\|_{L^{2}}^{\frac{1}{2}}\|g\|_{H^{2}}^{\frac{1}{2}} \quad \forall g \in H^{2}\left(\mathbb{T}^{2}\right) .
$$

Lemma 11 (Brézis-Gallouet inequality [5]). When $d=2$, for any $g \in H^{2}\left(\mathbb{T}^{2}\right)$, it holds that

$$
\|g\|_{L^{\infty}} \leq C\|g\|_{H^{1}} \sqrt{\ln \left(1+\|g\|_{H^{2}}\right)}+C\|g\|_{H^{1}} .
$$

Now we state the main result of this section.

Proposition 12. Let $d=2$ and

$$
\mathcal{A}(t) \stackrel{\text { def }}{=}\|\nabla u(t)\|_{L^{2}}^{2}+\lambda\|H(Q(t))\|_{L^{2}}^{2} .
$$

For any $\xi \in \mathbb{R}$, the following energy inequality holds:

$$
\begin{aligned}
& (3.6) \frac{d}{d t} \mathcal{A}(t)+\frac{\nu}{2}\|\Delta u(t)\|_{L^{2}}^{2}+\frac{\lambda \Gamma}{2}\|\nabla H(Q(t))\|_{L^{2}}^{2} \\
& \quad \leq C_{*}\{1+|\xi|[1+\ln (e+\ln (e+\mathcal{A}(t)))](e+\ln (e+\mathcal{A}(t)))\}[e+\mathcal{A}(t)] \mathcal{A}(t),
\end{aligned}
$$

where $C_{*}>0$ is a constant that depends on $\nu, \Gamma, L, \lambda, a, c, \mathbb{T}^{2},\left\|u_{0}\right\|_{L^{2}},\left\|Q_{0}\right\|_{H^{1}}$, and $\xi$.

Proof. After a lengthy calculation (see the appendix for details), we obtain

$$
\begin{aligned}
& \frac{1}{2} \frac{d}{d t} \mathcal{A}(t)+\nu\|\Delta u\|_{L^{2}}^{2}+\lambda \Gamma\|\nabla H(Q)\|_{L^{2}}^{2} \\
& =\int_{\mathbb{T}^{2}}(u \cdot \nabla u) \cdot \Delta u d x-2 \lambda \int_{\mathbb{T}^{2}} \nabla_{l} u_{k} \nabla_{l} \nabla_{k} Q_{i j} H_{i j} d x \\
& \quad+\frac{\lambda}{L} \int_{\mathbb{T}^{2}} u_{k} \nabla_{k} F_{i j} H_{i j} d x \\
& \quad-2 \lambda \int_{\mathbb{T}^{2}} \nabla_{j} u_{i}\left(\nabla_{l} Q_{k j} \nabla_{l} H_{i k}-\lambda \nabla_{l} Q_{i k} \nabla_{l} H_{k j}\right) d x \\
& \quad-\lambda \int_{\mathbb{T}^{2}} \nabla_{j} u_{i}\left(\Delta Q_{k j} H_{i k}-\Delta Q_{i k} H_{k j}\right) d x \\
& \quad+\lambda \xi \int_{\mathbb{T}^{2}}(D \Delta Q+\Delta Q D): H(Q) d x+4 \lambda \xi \int_{\mathbb{T}^{2}} \nabla_{l} D_{i k} \nabla_{l} Q_{k j} H_{i j} d x
\end{aligned}
$$

Copyright $@$ by SIAM. Unauthorized reproduction of this article is prohibited. 


$$
\begin{aligned}
& -2 \lambda \xi \int_{\mathbb{T}^{2}} \Delta\left(Q_{k l} Q_{j i}\right) \nabla_{j} u_{i} H_{k l} d x \\
& -4 \lambda \xi \int_{\mathbb{T}^{2}} \nabla_{m}\left(Q_{k l} Q_{j i}\right) \nabla_{m} \nabla_{j} u_{i} H_{k l} d x \\
& -\lambda \int_{\mathbb{T}^{2}} \frac{\partial F(Q)}{\partial Q}(u \cdot \nabla Q): H(Q) d x+\lambda \int_{\mathbb{T}^{2}} \frac{\partial F(Q)}{\partial Q} S(\nabla u, Q): H(Q) d x \\
& +\lambda \Gamma \int_{\mathbb{T}^{2}} \frac{\partial F(Q)}{\partial Q} H(Q): H(Q) d x \\
& \stackrel{\text { def }}{=} \sum_{i=1}^{12} J_{i},
\end{aligned}
$$

where $F(Q)=-a Q-c Q \operatorname{tr}\left(Q^{2}\right)$, since now we consider the $2 \mathrm{D}$ case (see (2.12) and $(6.3))$.

Below we shall estimate the terms $J_{1}$ through $J_{12}$ in (3.7). Let us take $\epsilon \in(0,1)$ to be a small constant that will be determined later.

The term $J_{1}$ can be easily estimated by using the Gagliardo-Nirenberg inequality (3.1) and the lower-order estimate (2.5):

$$
\begin{aligned}
J_{1} & \leq\|u\|_{L^{4}}\|\nabla u\|_{L^{4}}\|\Delta u\|_{L^{2}} \\
& \leq C\|u\|_{L^{2}}^{\frac{1}{2}}\|\nabla u\|_{L^{2}}\|\Delta u\|_{L^{2}}^{\frac{3}{2}} \\
& \leq \epsilon\|\Delta u\|_{L^{2}}^{2}+C\|\nabla u\|_{L^{2}}^{4} .
\end{aligned}
$$

Recalling (2.13) and using again (2.5), we observe that

$$
\begin{aligned}
\|\Delta Q\|_{L^{2}} & \leq \frac{1}{L}\|H(Q)\|_{L^{2}}+\frac{1}{L}\left\|a Q+c \operatorname{tr}\left(Q^{2}\right) Q\right\|_{L^{2}} \\
& \leq \frac{1}{L}\|H(Q)\|_{L^{2}}+C\left(\|Q\|_{H^{1}}\right) \\
& \leq \frac{1}{L}\|H(Q)\|_{L^{2}}+C .
\end{aligned}
$$

Meanwhile, applying (3.2) and (3.3) once more, we get

$$
\begin{aligned}
\|\nabla \Delta Q\|_{L^{2}} & \leq \frac{1}{L}\|\nabla H(Q)\|_{L^{2}}+\frac{1}{L}\left\|a \nabla Q+c \nabla\left[\operatorname{tr}\left(Q^{2}\right) Q\right]\right\|_{L^{2}} \\
& \leq \frac{1}{L}\|\nabla H(Q)\|_{L^{2}}+C\left(1+\|Q\|_{L^{\infty}}^{2}\right)\|\nabla Q\|_{L^{2}} \\
& \leq \frac{1}{L}\|\nabla H(Q)\|_{L^{2}}+C\left(1+\|\Delta Q\|_{L^{2}}\right) \\
& \leq \frac{1}{L}\|\nabla H(Q)\|_{L^{2}}+C\left(1+\|\nabla Q\|_{L^{2}}^{\frac{1}{2}}\|\nabla \Delta Q\|_{L^{2}}^{\frac{1}{2}}+\|\nabla Q\|_{L^{2}}\right) \\
& \leq \frac{1}{L}\|\nabla H(Q)\|_{L^{2}}+\frac{1}{2}\|\nabla \Delta Q\|_{L^{2}}+C,
\end{aligned}
$$

which implies

$$
\|\nabla \Delta Q\|_{L^{2}} \leq \frac{2}{L}\|\nabla H(Q)\|_{L^{2}}+C
$$

On the other hand, we infer from Agmon's inequality (3.3) and the estimates (2.5), (3.8) that

$$
\|Q\|_{L^{\infty}} \leq C\|Q\|_{L^{2}}^{\frac{1}{2}}\|Q\|_{H^{2}}^{\frac{1}{2}} \leq C\left(1+\|\Delta Q\|_{L^{2}}^{\frac{1}{2}}\right) \leq C\left(1+\|H(Q)\|_{L^{2}}^{\frac{1}{2}}\right) .
$$

Copyright $@$ by SIAM. Unauthorized reproduction of this article is prohibited. 
As a consequence, it follows from the Hölder inequality, the Gagliardo-Nirenberg inequality (3.1), and Young's inequality that

$$
\begin{aligned}
J_{2} \leq & C\|\nabla u\|_{L^{2}}\|Q\|_{W^{2,4}}\|H\|_{L^{4}} \\
\leq & C\|\nabla u\|_{L^{2}}\|Q\|_{H^{2}}^{\frac{1}{2}}\|Q\|_{H^{3}}^{\frac{1}{2}}\|H\|_{L^{2}}^{\frac{1}{2}}\|H\|_{H^{1}}^{\frac{1}{2}} \\
\leq & C\|\nabla u\|_{L^{2}}\left(\|\Delta Q\|_{L^{2}}^{\frac{1}{2}}\|\nabla \Delta Q\|_{L^{2}}^{\frac{1}{2}}+\|\Delta Q\|_{L^{2}}+1\right)\left(\|H\|_{L^{2}}^{\frac{1}{2}}\|\nabla H\|_{L^{2}}^{\frac{1}{2}}+\|H\|_{L^{2}}\right) \\
\leq & C\|\nabla u\|_{L^{2}}\left(\|H\|_{L^{2}}^{\frac{1}{2}}\|\nabla H\|_{L^{2}}^{\frac{1}{2}}+\|\nabla H\|_{L^{2}}^{\frac{1}{2}}+\|H\|_{L^{2}}+1\right) \\
& \times\left(\|H\|_{L^{2}}^{\frac{1}{2}}\|\nabla H\|_{L^{2}}^{\frac{1}{2}}+\|H\|_{L^{2}}\right) \\
\leq & \epsilon\|\nabla H\|_{L^{2}}^{2}+C\|\nabla u\|_{L^{2}}^{2}+C\|\nabla u\|_{L^{2}}^{4}+C\|H\|_{L^{2}}^{2}+C\|H\|_{L^{2}}^{4} \\
\leq & \epsilon\|\nabla H\|_{L^{2}}^{2}+C \mathcal{A}(1+\mathcal{A}) .
\end{aligned}
$$

For $J_{3}$, using the inequalities (3.1) and (3.3), we obtain that

$$
\begin{aligned}
J_{3} & \leq\|u\|_{L^{4}}\|\nabla Q\|_{L^{4}}\left(1+\|Q\|_{L^{\infty}}^{2}\right)\|H\|_{L^{2}} \\
& \leq C\|\nabla u\|_{L^{2}}\|\Delta Q\|_{L^{2}}^{\frac{1}{2}}\|\nabla Q\|_{L^{2}}^{\frac{1}{2}}\left(1+\|\Delta Q\|_{L^{2}}\right)\|H\|_{L^{2}} \\
& \leq C\|\nabla u\|_{L^{2}}\left(1+\|\nabla \Delta Q\|_{L^{2}}^{\frac{1}{2}}\|\nabla Q\|_{L^{2}}^{\frac{1}{2}}\right)\|\Delta Q\|_{L^{2}}^{\frac{1}{2}}\|H\|_{L^{2}} \\
& \leq \epsilon\|\nabla H\|_{L^{2}}^{2}+C\|\nabla u\|_{L^{2}}^{4}+C\|H\|_{L^{2}}^{4}+C\|H\|_{L^{2}}^{2} \\
& \leq \epsilon\|\nabla H\|_{L^{2}}^{2}+C \mathcal{A}(1+\mathcal{A}) .
\end{aligned}
$$

The terms $J_{4}$ and $J_{5}$ can be estimated as

$$
\begin{aligned}
J_{4} & \leq C\|\nabla u\|_{L^{4}}\|\nabla Q\|_{L^{4}}\|\nabla H\|_{L^{2}} \\
& \leq C\|\nabla u\|_{L^{2}}^{\frac{1}{2}}\|\Delta u\|_{L^{2}}^{\frac{1}{2}}\|\nabla Q\|_{L^{2}}^{\frac{1}{2}}\|\Delta Q\|_{L^{2}}^{\frac{1}{2}}\|\nabla H\|_{L^{2}} \\
& \leq \epsilon\|\Delta u\|_{L^{2}}^{2}+\epsilon\|\nabla H\|_{L^{2}}^{2}+C\|\nabla u\|_{L^{2}}^{2}+C\|\nabla u\|_{L^{2}}^{2}\|H\|_{L^{2}}^{2} \\
& \leq \epsilon\|\Delta u\|_{L^{2}}^{2}+\epsilon\|\nabla H\|_{L^{2}}^{2}+C \mathcal{A}(1+\mathcal{A}), \\
J_{5} & \leq C\|\nabla u\|_{L^{4}}\|\Delta Q\|_{L^{2}}\|H\|_{L^{4}} \\
& \leq C\|\nabla u\|_{L^{2}}^{\frac{1}{2}}\|\Delta u\|_{L^{2}}^{\frac{1}{2}}\left(1+\|H\|_{L^{2}}\right)\left(\|H\|_{L^{2}}^{\frac{1}{2}}\|\nabla H\|_{L^{2}}^{\frac{1}{2}}+\|H\|_{L^{2}}\right) \\
& \leq \epsilon\|\Delta u\|_{L^{2}}^{2}+\epsilon\|\nabla H\|_{L^{2}}^{2}+C\|\nabla u\|_{L^{2}}^{2}+C\|\nabla u\|_{L^{2}}^{4}+C\|H\|_{L^{2}}^{2}+C\|H\|_{L^{2}}^{4} \\
& \leq \epsilon\|\Delta u\|_{L^{2}}^{2}+\epsilon\|\nabla H\|_{L^{2}}^{2}+C \mathcal{A}(1+\mathcal{A}) .
\end{aligned}
$$

Besides, for $J_{10}$ and $J_{12}$ the following inequality holds:

$$
\begin{aligned}
J_{10} & +J_{12} \\
& \leq C\left(1+\|Q\|_{L^{\infty}}^{2}\right)\left(\|H\|_{L^{2}}+\|u\|_{L^{\infty}}\|\nabla Q\|_{L^{2}}\right)\|H\|_{L^{2}} \\
& \leq C\left(1+\|H\|_{L^{2}}\right)\left(\|H\|_{L^{2}}+\|u\|_{L^{2}}^{\frac{1}{2}}\|\Delta u\|_{L^{2}}^{\frac{1}{2}}\right)\|H\|_{L^{2}} \\
& \leq C\left(1+\|H\|_{L^{2}}\right)\|H\|_{L^{2}}^{2}+C\|H\|_{L^{2}}^{2}\|u\|_{L^{2}}^{\frac{1}{2}}\|\Delta u\|_{L^{2}}^{\frac{1}{2}}+C\|H\|_{L^{2}}\|\nabla u\|_{L^{2}}^{\frac{1}{2}}\|\Delta u\|_{L^{2}}^{\frac{1}{2}} \\
& \leq \epsilon\|\Delta u\|_{L^{2}}^{2}+C\|\nabla u\|_{L^{2}}^{2}+C\|\nabla u\|_{L^{2}}^{4}+C\|H\|_{L^{2}}^{2}+C\|H\|_{L^{2}}^{4} \\
& \leq \epsilon\|\Delta u\|_{L^{2}}^{2}+C \mathcal{A}(1+\mathcal{A}) .
\end{aligned}
$$

It remains to estimate the terms $J_{6}, \ldots, J_{9}$ and $J_{11}$ involving the parameter $\xi$, which all vanish when $\xi=0$. Thus, we only need to consider the case $\xi \neq 0$ (with $\xi$ being arbitrary but fixed).

Copyright $\odot$ by SIAM. Unauthorized reproduction of this article is prohibited. 
The term $J_{6}$ can be estimated in the same way as for $J_{2}$, that is,

$$
\begin{aligned}
J_{6} & \leq C|\xi|\|\nabla u\|_{L^{2}}\|\Delta Q\|_{L^{4}}\|H\|_{L^{4}} \\
& \leq \epsilon\|\nabla H\|_{L^{2}}^{2}+C\left(1+|\xi|^{2}\right) \mathcal{A}(1+\mathcal{A}) .
\end{aligned}
$$

For $J_{7}$, using the Hölder inequality, (3.1), (3.8), and Young's inequality, we have

$$
\begin{aligned}
J_{7} & \leq C|\xi|\|\Delta u\|_{L^{2}}\|\nabla Q\|_{L^{4}}\|H\|_{L^{4}} \\
& \leq C|\xi|\|\Delta u\|_{L^{2}}\|\nabla Q\|_{L^{2}}^{\frac{1}{2}}\|\Delta Q\|_{L^{2}}^{\frac{1}{2}}\left(\|H\|_{L^{2}}^{\frac{1}{2}}\|\nabla H\|_{L^{2}}^{\frac{1}{2}}+\|H\|_{L^{2}}\right) \\
& \leq \epsilon\|\Delta u\|_{L^{2}}^{2}+\epsilon\|\nabla H\|_{L^{2}}^{2}+C\left(1+|\xi|^{4}\right)\left(\|H\|_{L^{2}}^{2}+\|H\|_{L^{2}}^{4}\right) \\
& \leq \epsilon\|\Delta u\|_{L^{2}}^{2}+\epsilon\|\nabla H\|_{L^{2}}^{2}+C\left(1+|\xi|^{4}\right) \mathcal{A}(1+\mathcal{A}) .
\end{aligned}
$$

Next, we treat $J_{11}$ and postpone the estimates for terms $J_{8}, J_{9}$ that are more involved.

$$
\begin{aligned}
J_{11} & \leq C\left(1+\|Q\|_{L^{\infty}}^{2}\right)\|S(\nabla u, Q)\|_{L^{2}}\|H\|_{L^{2}} \\
& \leq C\left(1+\|H\|_{L^{2}}\right)\left(1+\|Q\|_{L^{\infty}}\right)^{2}\|\nabla u\|_{L^{2}}\|H\|_{L^{2}} \\
& \leq C\left(1+\|H\|_{L^{2}}\right)^{2}\|\nabla u\|_{L^{2}}\|H\|_{L^{2}} \\
& \leq C\|\nabla u\|_{L^{2}}\|H\|_{L^{2}}+C\|H\|_{L^{2}}^{3}\|\Delta u\|_{L^{2}}^{\frac{1}{2}}\|u\|_{L^{2}}^{\frac{1}{2}} \\
& \leq \epsilon\|\Delta u\|_{L^{2}}^{2}+C\|\nabla u\|_{L^{2}}^{2}+C\|H\|_{L^{2}}^{2}+C\|H\|_{L^{2}}^{4} \\
& \leq \epsilon\|\Delta u\|_{L^{2}}^{2}+C \mathcal{A}(1+\mathcal{A}) .
\end{aligned}
$$

Now let us consider the term $J_{8}$. By a similar argument for $J_{5}$ and using the BrézisGallouet inequality (3.4), we obtain that

$$
\begin{aligned}
J_{8} \leq & 2|\xi|\|\nabla u\|_{L^{4}}\|H\|_{L^{4}}\|\Delta(Q Q)\|_{L^{2}} \\
\leq & C|\xi|\|\nabla u\|_{L^{4}}\|H\|_{L^{4}}\left(\|Q\|_{L^{\infty}}\|\Delta Q\|_{L^{2}}+\|\nabla Q\|_{L^{4}}^{2}\right) \\
\leq & C|\xi|\|\nabla u\|_{L^{4}}\|H\|_{L^{4}}\left(\|Q\|_{L^{\infty}}\|\Delta Q\|_{L^{2}}+\|\Delta Q\|_{L^{2}}\|\nabla Q\|_{L^{2}}\right) \\
\leq & C|\xi|\|\nabla u\|_{L^{2}}^{\frac{1}{2}}\|\Delta u\|_{L^{2}}^{\frac{1}{2}}\left(\|H\|_{L^{2}}^{\frac{1}{2}}\|\nabla H\|_{L^{2}}^{\frac{1}{2}}+\|H\|_{L^{2}}\right)\left(1+\|H\|_{L^{2}}\right)\left(1+\|Q\|_{L^{\infty}}\right) \\
\leq & \epsilon\|\Delta u\|^{2}+\epsilon\|\nabla H\|^{2} \\
& +C\left(|\xi|+\left.|\xi|\right|^{4}\right)\left(1+\|Q\|_{L^{\infty}}^{2}\right)\left(\|\nabla u\|_{L^{2}}^{4}+\|\nabla u\|_{L^{2}}^{2}+\|H\|_{L^{2}}^{4}+\|H\|_{L^{2}}^{2}\right) \\
\leq & \epsilon\|\Delta u\|^{2}+\epsilon\|\nabla H\|^{2}+C\left(|\xi|+|\xi|^{4}\right) \mathcal{B A}(1+\mathcal{A})
\end{aligned}
$$

where we have set

$$
\mathcal{B} \stackrel{\text { def }}{=} e+\ln (e+\mathcal{A})>e .
$$

Concerning the last term $J_{9}$, by the Hölder inequality we have, for any $p \in(0,1)$,

$$
J_{9} \leq 4|\xi|\|Q\|_{L^{\infty}}\|\nabla Q\|_{L^{\frac{2}{p}}}\left\|\nabla^{2} u\right\|_{L^{2}}\|H\|_{L^{\frac{2}{1-p}}} .
$$

For any $p \in\left(0, \frac{1}{2}\right)$, applying the $L^{p}$-interpolation inequality Lemma 9 with $\eta=p^{-1}>$ 1 and $\eta=(1-p)^{-1} \in(1,2)$, respectively, we deduce that

$$
\begin{aligned}
\|\nabla Q\|_{L^{\frac{2}{p}}} & \leq C \sqrt{p^{-1}}\|Q\|_{H^{2}}^{1-p}\|\nabla Q\|_{L^{2}}^{p} \\
& \leq C \sqrt{p^{-1}}\|H\|_{L^{2}}^{1-p}\|\nabla Q\|_{L^{2}}^{p}+C \sqrt{p^{-1}} \\
& \leq C \sqrt{p^{-1}}\|H\|_{L^{2}}^{1-p}+C \sqrt{p^{-1}}
\end{aligned}
$$

Copyright $\odot$ by SIAM. Unauthorized reproduction of this article is prohibited. 
and

$$
\begin{aligned}
\|H\|_{L^{\frac{2}{1-p}}} & \leq C \sqrt{(1-p)^{-1}}\|H\|_{H^{1}}^{p}\|H\|_{L^{2}}^{1-p} \\
& \leq C \sqrt{(1-p)^{-1}}\|\nabla H\|_{L^{2}}^{p}\|H\|_{L^{2}}^{1-p}+C \sqrt{(1-p)^{-1}}\|H\|_{L^{2}} \\
& \leq C\|\nabla H\|_{L^{2}}^{p}\|H\|_{L^{2}}^{1-p}+C\|H\|_{L^{2}} .
\end{aligned}
$$

Hence, by the Brézis-Gallouet inequality (3.4), estimates (3.12)-(3.14), and Young's inequality, we have

$$
\begin{aligned}
J_{9} \leq & C|\xi| \sqrt{p^{-1}}\|\Delta u\|_{L^{2}}\|Q\|_{L^{\infty}} \\
& \times\left(\|\nabla H\|_{L^{2}}^{p}\|H\|_{L^{2}}^{2(1-p)}+\|H\|_{L^{2}}^{2-p}+\|\nabla H\|_{L^{2}}^{p}\|H\|_{L^{2}}^{1-p}+\|H\|_{L^{2}}\right) \\
\leq & \epsilon|\xi|\|\Delta u\|_{L^{2}}^{2}+\epsilon|\xi|\|\nabla H\|_{L^{2}}^{2}+C|\xi| p^{-\frac{1}{1-p}}\|Q\|_{L^{\infty}}^{\frac{2}{1-p}}\left(\|H\|_{L^{2}}^{4}+\|H\|_{L^{2}}^{2}\right) \\
& +C|\xi| p^{-1}\|Q\|_{L^{\infty}}^{2}\left(\|H\|_{L^{2}}^{4}+\|H\|_{L^{2}}^{2}\right) \\
\leq & \epsilon|\xi|\|\Delta u\|_{L^{2}}^{2}+\epsilon|\xi|\|\nabla H\|_{L^{2}}^{2} \\
& +C|\xi| p^{-\frac{1}{1-p}}[e+\ln (e+\mathcal{A})]^{\frac{1}{1-p}}\left(\|H\|_{L^{2}}^{4}+\|H\|_{L^{2}}^{2}\right) \\
& +C|\xi| p^{-1}[e+\ln (e+\mathcal{A})]\left(\|H\|_{L^{2}}^{4}+\|H\|_{L^{2}}^{2}\right) \\
\stackrel{\text { def }}{=} & \epsilon|\xi|\|\Delta u\|_{L^{2}}^{2}+\epsilon|\xi|\|\nabla H\|_{L^{2}}^{2}+J_{9 a}+J_{9 b} \quad \forall p \in\left(0, \frac{1}{2}\right) .
\end{aligned}
$$

Since the constant $C$ in the estimate (3.15) is independent of the parameter $p \in\left(0, \frac{1}{2}\right)$, in the spirit of [31] we can take the exponent

$$
p=(1+\ln \mathcal{B})^{-1},
$$

where $\mathcal{B}$ is defined in (3.11). We note that with this choice $p$ may not be a constant, but it is always true that $p \in\left(0, \frac{1}{2}\right)$. Then it follows from (3.15) that

$$
J_{9 b} \leq C|\xi|(1+\ln \mathcal{B}) \mathcal{B} \mathcal{A}(1+\mathcal{A})
$$

and

$$
\begin{aligned}
J_{9 a} & \leq C|\xi| p^{-\frac{1}{1-p}}[e+\ln (e+\mathcal{A})]^{\frac{1}{1-p}}\left(\|H\|_{L^{2}}^{4}+\|H\|_{L^{2}}^{2}\right) \\
& \leq C|\xi|[(1+\ln \mathcal{B}) \mathcal{B}]^{1+\frac{1}{\ln B}} \mathcal{A}(1+\mathcal{A}) \\
& \leq C|\xi|(1+\ln \mathcal{B}) \mathcal{B} \mathcal{A}(1+\mathcal{A})
\end{aligned}
$$

where we have used the simple fact that the quantity

$$
[(1+\ln \mathcal{B}) \mathcal{B}]^{\frac{1}{\ln B}}=\left[(1+\ln \mathcal{B}) e^{\ln \mathcal{B}}\right]^{\frac{1}{\ln \mathcal{B}}}=e(1+\ln \mathcal{B})^{\frac{1}{\ln \mathcal{B}}}
$$

is uniformly bounded for all $\mathcal{B}>e$. As a consequence of (3.15)-(3.17), we deduce that

$$
J_{9} \leq \epsilon|\xi|\|\Delta u\|_{L^{2}}^{2}+\epsilon|\xi|\|\nabla H\|_{L^{2}}^{2}+C|\xi|(1+\ln \mathcal{B}) \mathcal{B A}(1+\mathcal{A}) .
$$

Copyright $@$ ㅇ by SIAM. Unauthorized reproduction of this article is prohibited. 
At last, we take the small constant $\epsilon$ such that

$$
\epsilon \in\left(0, \frac{\min \{\nu, \lambda \Gamma\}}{2(7+|\xi|)}\right) .
$$

From (3.7) and the above estimates for terms $J_{1}, \ldots, J_{12}$, it follows that

$$
\frac{d}{d t} \mathcal{A}+\frac{\nu}{2}\|\Delta u\|_{L^{2}}^{2}+\frac{\lambda \Gamma}{2}\|\nabla H\|_{L^{2}}^{2} \leq C_{*}[1+|\xi|(1+\ln \mathcal{B}) \mathcal{B}] \mathcal{A}(1+\mathcal{A}),
$$

which easily implies the conclusion (3.6). The proof is complete.

Remark 13. If $\xi=0$, the inequality (3.6) reduces to

$$
\frac{d}{d t} \mathcal{A}(t) \leq C_{*}[e+\mathcal{A}(t)] \mathcal{A}(t)
$$

which is the same as the higher-order energy inequality derived in [2, Lemma 7.1].

4. Global strong solutions in $\mathbf{2 D}$. In this section, we show that starting from initial data with higher regularity, the problem (1.1)-(1.5) admits a unique global strong solution.

4.1. Semi-Galerkin approximation scheme. Inspired by [25] on the simplified Ericksen-Leslie system for incompressible nematic liquid crystal flow, we can work with a semi-Galerkin scheme in the periodic setting. For the convenience of readers, we briefly describe it below. Recalling the classical spectral theorem for compact operators in Hilbert spaces and standard results for the stationary Stokes system, we have the following results on eigenfunctions of the Stokes operator $\mathcal{S}$ for $u$. Let $\left\{v_{n}\right\}_{n=1}^{\infty}$ be the eigenvectors of the Stokes operator $\mathcal{S}$ in the torus $\mathbb{T}^{2}$ with zero mean,

$$
\begin{gathered}
\mathcal{S} v_{n}=\kappa_{n} v_{n}, \quad \nabla \cdot v_{n}=0, \quad \int_{\mathbb{T}^{2}} v_{n}(x) d x=0, \quad \text { in } \mathbb{T}^{2}, \\
v_{n}\left(x+e_{i}\right)=v_{n}(x), \quad x \in \mathbb{T}^{2},
\end{gathered}
$$

where $0<\kappa_{1} \leq \kappa_{2} \leq \cdots \nearrow+\infty$ are corresponding eigenvalues. The eigenvectors $v_{n}$ are smooth, and the sequence $\left\{v_{n}\right\}_{n=1}^{\infty}$ forms an orthogonal basis of $\mathbf{H}$ as well as $\mathbf{V}$ (see, e.g., [36]).

Taking an arbitrary but fixed integer $N \in \mathbb{N}$, we consider the finite-dimensional space $V_{N}=\operatorname{span}\left\{v_{n}\right\}_{n=1}^{N}$ along with the orthogonal projection operators $\Pi_{N}: \mathbf{H} \rightarrow$ $V_{N}$, which are bounded linear operators with norms bounded by one. For any $T>0$, we seek approximations of solutions to the problem (1.1)-(1.5). The approximation of velocity $u^{N}$ takes the form

$$
u^{N}=\sum_{i=1}^{N} h_{i}(t) v_{i}(x),
$$

which solves

$$
\begin{aligned}
& \int_{\mathbb{T}^{2}}\left(u^{N}\right)_{t} \cdot v_{k} d x d t-\int_{\mathbb{T}^{2}}\left(u^{N} \otimes u^{N}\right): \nabla v_{k} d x+\nu \int_{\mathbb{T}^{2}} \nabla u^{N}: \nabla v_{k} d x \\
& \quad=-\int_{\mathbb{T}^{2}}\left(\sigma^{N}+\tau^{N}\right): \nabla v_{k} d x \quad \forall t \in(0, T)
\end{aligned}
$$

Copyright $\odot$ by SIAM. Unauthorized reproduction of this article is prohibited. 
for any $k=1, \ldots, N$. In (4.1), the approximations of stress tensors are given by

$$
\begin{aligned}
& \tau^{N} \stackrel{\text { def }}{=}-\xi\left(Q^{N}+\frac{1}{2} \mathbb{I}\right) H^{N}\left(Q^{N}\right)-\xi H^{N}\left(Q^{N}\right)\left(Q^{N}+\frac{1}{2} \mathbb{I}\right) \\
&+2 \xi\left(Q^{N}+\frac{1}{2} \mathbb{I}\right) \operatorname{tr}\left(Q^{N} H^{N}\left(Q^{N}\right)\right)-L \nabla Q^{N} \odot \nabla Q^{N}, \\
& \sigma^{N} \stackrel{\text { def }}{=} Q^{N} H^{N}\left(Q^{N}\right)-H^{N}\left(Q^{N}\right) Q^{N},
\end{aligned}
$$

where

$$
H^{N}\left(Q^{N}\right) \stackrel{\text { def }}{=} L \Delta Q^{N}-a Q^{N}-c Q^{N} \operatorname{tr}\left(\left(Q^{N}\right)^{2}\right) .
$$

On the other hand, the approximate function $Q^{N}$ is determined in terms of $u^{N}$ as the unique solution to the parabolic system

$$
Q_{t}^{N}+u^{N} \cdot \nabla Q^{N}-S\left(\nabla u^{N}, Q^{N}\right)=\Gamma H^{N}\left(Q^{N}\right), \quad(x, t) \in \mathbb{T}^{2} \times \mathbb{R}^{+},
$$

where

$$
\begin{aligned}
S^{N}\left(\nabla u^{N}, Q^{N}\right) \stackrel{\text { def }}{=} & \left(\xi D^{N}+\Omega^{N}\right)\left(Q^{N}+\frac{1}{2} \mathbb{I}\right)+\left(Q^{N}+\frac{1}{2} \mathbb{I}\right)\left(\xi D^{N}-\Omega^{N}\right) \\
& -2 \xi\left(Q^{N}+\frac{1}{2} \mathbb{I}\right) \operatorname{tr}\left(Q^{N} \nabla u^{N}\right)
\end{aligned}
$$

with

$$
D^{N}=\frac{\nabla u^{N}+\nabla^{T} u^{N}}{2}, \quad \Omega^{N}=\frac{\nabla u^{N}-\nabla^{T} u^{N}}{2} .
$$

The initial conditions are given by

$$
\left.u^{N}\right|_{t=0}=\Pi_{N} u_{0},\left.\quad Q^{N}\right|_{t=0}=Q_{0}, \quad x \in \mathbb{T}^{2} .
$$

4.2. Proof of Theorem 6. The proof for the existence of global strong solutions consists of several steps.

Existence of approximate solutions. For any fixed integer $N$, we have the following result on local existence of the approximate solution $\left(u^{N}, Q^{N}\right)$.

Proposition 14. Suppose $u_{0} \in \mathbf{V}, Q_{0} \in H^{2}\left(\mathbb{T}^{2}, S_{0}^{(2)}\right)$. For any $N \in \mathbb{N}$, there exists a $T_{N}>0$ depending on $\left\|u_{0}\right\|_{H^{1}},\left\|Q_{0}\right\|_{H^{2}}$, and $N$ such that the approximate problem (4.1)-(4.7) admits a solution $\left(u^{N}, Q^{N}\right)$ satisfying

$$
\begin{aligned}
u^{N} \in & L^{\infty}\left(0, T_{N} ; \mathbf{V}\right) \cap L^{2}\left(0, T_{N} ; H^{2}\left(\mathbb{T}^{2}, \mathbb{R}^{2}\right)\right) \cap H^{1}\left(0, T_{N} ; \mathbf{H}\right), \\
Q^{N} \in & L^{\infty}\left(0, T_{N} ; H^{2}\left(\mathbb{T}^{2}, S_{0}^{(2)}\right)\right) \cap L^{2}\left(0, T_{N} ; H^{3}\left(\mathbb{T}^{2}, S_{0}^{(2)}\right)\right) \\
& \cap H^{1}\left(0, T_{N} ; H^{1}\left(\mathbb{T}^{2}, S_{0}^{(2)}\right)\right) .
\end{aligned}
$$

Proposition 14 can be proved by a classical Schauder's argument (see, e.g., [25]). Indeed, given a vector $\tilde{u} \in C\left([0, T] ; V_{N}\right)$, we find a $Q=Q[\tilde{u}]$ by solving (4.5) with $u^{N}$ replaced by $\tilde{u}$. Inserting $Q[\tilde{u}]$ back into (4.1), the solution $u$ to the resulting ODE system defines a continuous mapping $\mathcal{T}: \tilde{u} \mapsto \mathcal{T}[\tilde{u}]=u$. It is standard to show that $\mathcal{T}$ admits a fixed point by means of the classical Schauder's argument on $\left(0, T_{N}\right)$ with certain $T_{N}>0$ depending on $\left\|u_{0}\right\|_{H^{1}},\left\|Q_{0}\right\|_{H^{2}}$, and $N$. We leave the detailed proof to interested readers.

Copyright $@$ by SIAM. Unauthorized reproduction of this article is prohibited. 
Remark 15. Since we are working in the periodic domain $\mathbb{T}^{2}$, by the classical regularity theory for parabolic equations (cf. [23]) and a bootstrap argument, we can see that $\left(u^{N}, Q^{N}\right)$ is $C^{\infty}$ in the interior of $\mathbb{T}^{2} \times\left(0, T_{N}\right)$.

In order to prove the existence of global strong solutions, we need to derive some uniform estimates for approximate solutions $\left(u^{N}, Q^{N}\right)$, which are independent of the approximation parameter $N$ as well as the time $t$.

Lower-order estimates. A similar argument like in [1] yields that the approximate solutions satisfy the following energy identity:

$$
\frac{d}{d t} \mathcal{E}^{N}(t)=-\nu \int_{\mathbb{T}^{2}}\left|\nabla u^{N}\right|^{2} d x-\lambda \Gamma \int_{\mathbb{T}^{2}}\left|H^{N}\left(Q^{N}\right)\right|^{2} d x \leq 0 \quad \forall t \in\left[0, T_{N}\right),
$$

where

$$
\mathcal{E}^{N}(t) \stackrel{\text { def }}{=} \frac{1}{2} \int_{\mathbb{T}^{2}}\left|u^{N}\right|^{2}(x, t) d x+\lambda \mathcal{F}\left(Q^{N}(t)\right) .
$$

As in Lemma 2, the energy identity (4.8) provides uniform estimates for $u^{N}$ and $Q^{N}$ such that

$$
\begin{aligned}
\left\|u^{N}(t)\right\|_{L^{2}}+\left\|Q^{N}(t)\right\|_{H^{1}} & \leq C & & \forall t \in\left[0, T_{N}\right), \\
\int_{0}^{t} \int_{\mathbb{T}^{d}}\left|\nabla u^{N}(\tau)\right|^{2}+\left|\Delta Q^{N}(\tau)\right|^{2} d x d \tau & \leq C(1+t) & & \forall t \in\left[0, T_{N}\right),
\end{aligned}
$$

where the constant $C>0$ depends on $\left\|u_{0}\right\|,\left\|Q_{0}\right\|_{H^{1}}, L, \lambda, \nu, \Gamma, a, c$, and $\mathbb{T}^{2}$ but is independent of the parameter $N$ and time $t$.

Higher-order estimates. It is easy to see that the calculations we made in section 3 for smooth solutions $(u, Q)$ to the problem (1.1)-(1.5) still hold for the approximate solutions $\left(u^{N}, Q^{N}\right)$. Thus, for $\left(u^{N}, Q^{N}\right)$, we introduce the quantity

$$
\mathcal{A}_{N}(t) \stackrel{\text { def }}{=}\left\|\nabla u^{N}(t)\right\|_{L^{2}}^{2}+\lambda\left\|H^{N}\left(Q^{N}(t)\right)\right\|_{L^{2}}^{2} .
$$

In particular, we infer from (4.8) that

$$
\int_{0}^{t} \int_{\mathbb{T}^{2}} \nu\left|\nabla u^{N}(\tau)\right|^{2}+\lambda \Gamma\left|H^{N}\left(Q^{N}(\tau)\right)\right|^{2} d x d \tau \leq K \quad \forall t \in\left[0, T_{N}\right),
$$

where $K>0$ is a constant that only depends on $\left\|u_{0}\right\|_{L^{2}},\left\|Q_{0}\right\|_{H^{1}}, L, \lambda, a, c, \mathbb{T}^{2}$. Then we have

$$
\int_{0}^{t} \mathcal{A}_{N}(\tau) d \tau \leq \frac{K}{\min \{\nu, \Gamma\}} \quad \forall t \in\left[0, T_{N}\right)
$$

On the other hand, using the lower-order estimate (4.10), for any $\xi \in \mathbb{R}$, we can get the following higher-order energy inequality for all $t \in\left[0, T_{N}\right)$ :

$$
\begin{aligned}
\frac{d}{d t} \mathcal{A}_{N}(t)+\frac{\nu}{2}\left\|\Delta u^{N}\right\|_{L^{2}}^{2}+\frac{\lambda \Gamma}{2}\left\|\nabla H^{N}\left(Q^{N}\right)\right\|_{L^{2}}^{2} \\
\leq C_{*}\left\{1+|\xi|\left[1+\ln \left(e+\ln \left(e+\mathcal{A}_{N}(t)\right)\right)\right]\left[e+\ln \left(e+\mathcal{A}_{N}(t)\right)\right]\right\} \\
\quad \times\left[e+\mathcal{A}_{N}(t)\right] \mathcal{A}_{N}(t),
\end{aligned}
$$

where $C_{*}>0$ is a constant that depends on $\nu, \Gamma, L, \lambda, a, c, \mathbb{T}^{2},\left\|u_{0}\right\|_{L^{2}},\left\|Q_{0}\right\|_{H^{1}}$, and $\xi$ but is independent of $N$ and $t$. 
We consider two cases.

Case 1. If $\xi=0$, then it follows from (4.15) that

$$
\frac{d}{d t} \ln \left[e+\mathcal{A}_{N}(t)\right] \leq C_{*} \mathcal{A}_{N}(t)
$$

which implies

$$
\begin{aligned}
\mathcal{A}_{N}(t) & \leq\left[e+\mathcal{A}_{N}(0)\right] e^{C_{*} \int_{0}^{t} \mathcal{A}_{N}(\tau) d \tau} \\
& \leq\left[e+\mathcal{A}_{N}(0)\right] e^{\frac{C_{*} K}{\min \{\nu, \Gamma\}}} \\
& \leq C \quad \forall t \in\left[0, T_{N}\right),
\end{aligned}
$$

where $C>0$ is a constant that depends on $\nu, \Gamma, L, \lambda, a, c, \mathbb{T}^{2},\left\|u_{0}\right\|_{\mathbf{V}},\left\|Q_{0}\right\|_{H^{2}}$.

Case 2. If $\xi \neq 0$, then we deduce from (4.15) that

$$
\frac{d}{d t} \ln \mathcal{Z}_{N}(t) \leq C_{*}(1+|\xi|) \mathcal{A}_{N}(t)
$$

where

$$
\mathcal{Z}_{N}(t) \stackrel{\text { def }}{=} 1+\ln \left(1+\ln \left[e+\mathcal{A}_{N}(t)\right]\right) .
$$

Integrating (4.16) with respect to time and using (4.14), we have

$$
\begin{aligned}
\ln \mathcal{Z}_{N}(t) & \leq \ln \mathcal{Z}_{N}(0)+C_{*}(1+|\xi|) \int_{0}^{t} \mathcal{A}_{N}(\tau) d \tau \\
& \leq \ln \mathcal{Z}_{N}(0)+\frac{\left(C_{*}\right)^{2} K(1+|\xi|)}{\min \{\nu, \Gamma\}} \quad \forall t \in\left[0, T_{N}\right),
\end{aligned}
$$

which again yields that

$$
\mathcal{A}_{N}(t) \leq C \quad \forall t \in\left[0, T_{N}\right)
$$

For both cases, after integrating the differential inequality (4.15) with respect to time, we obtain

$$
\int_{0}^{t}\left(\left\|\Delta u^{N}(\tau)\right\|_{L^{2}}^{2}+\left\|\nabla H^{N}\left(Q^{N}(\tau)\right)\right\|_{L^{2}}^{2}\right) d \tau \leq C \quad \forall t \in\left[0, T_{N}\right) .
$$

As a consequence, the following uniform higher-order estimates hold:

$$
\begin{array}{cc}
\left\|u^{N}(t)\right\|_{H^{1}}+\left\|Q^{N}(t)\right\|_{H^{2}} \leq C & \forall t \in\left[0, T_{N}\right), \\
\int_{0}^{t} \int_{\mathbb{T}^{2}}\left|\Delta u^{N}(\tau)\right|^{2}+\left|\nabla \Delta Q^{N}(\tau)\right|^{2} d x d \tau \leq C(1+t) & \forall t \in\left[0, T_{N}\right),
\end{array}
$$

where the constant $C>0$ depends on $\left\|u_{0}\right\|_{H^{1}},\left\|Q_{0}\right\|_{H^{2}}, L, \lambda, \nu, \Gamma, a, c, \xi$, and $\mathbb{T}^{2}$ but is independent of the parameter $N$ and the time $t$.

Passage to the limit as $N \rightarrow \infty$. First, we can deduce from the above uniformin-time lower-order and higher-order estimates (4.10), (4.19) that the approximate solutions $\left(u^{N}, Q^{N}\right)$ cannot blow up in finite time. Thus, for any $N \in \mathbb{N}$, it holds that 
$T_{N}=+\infty$ such that every approximate solution $\left(u^{N}, Q^{N}\right)$ can be extended to the time interval $[0, T]$ for arbitrary $T>0$.

Second, since the uniform estimates (4.10), (4.11), (4.19), (4.20) are also independent of the approximation parameter $N$, we infer from (4.1), (4.5), and the Hölder inequality that for any $T>0$ and $N \in \mathbb{N}$,

$$
\begin{aligned}
u^{N} & \in L^{\infty}(0, T ; \mathbf{V}) \cap L^{2}\left(0, T ; H^{2}\left(\mathbb{T}^{2}, \mathbb{R}^{2}\right)\right) \cap H^{1}(0, T ; \mathbf{H}), \\
Q^{N} & \in L^{\infty}\left(0, T ; H^{2}\left(\mathbb{T}^{2}, S_{0}^{(2)}\right)\right) \cap L^{2}\left(0, T ; H^{3}\left(\mathbb{T}^{2}, S_{0}^{(2)}\right)\right) \cap H^{1}\left(0, T ; H^{1}\left(\mathbb{T}^{2}, S_{0}^{(2)}\right)\right) .
\end{aligned}
$$

The above uniform estimates together with standard weak compactness results and the Aubin-Lions compactness lemma (see, e.g., [35, Corollary 4, section 8]) enable us to pass to the limit as $N \rightarrow \infty$ (up to a subsequence) to obtain a limit pair $(u, Q$ ), which turns out to be a global strong solution to the original Navier-Stokes and Qtensor system (1.1)-(1.5). Since the argument is standard (cf. [2]), we omit the details here.

Uniqueness. The uniqueness of strong solutions is a direct consequence of [31, section 5], where a weak-strong uniqueness result is given in $\mathbb{R}^{2}$.

Let $\left(u_{i}, Q_{i}\right), i=1,2$ be two global strong solutions of the problem (1.1)-(1.5) subject to initial data $\left(u_{i 0}, Q_{i 0}\right), i=1,2$, respectively. Since we are dealing with the periodic domain, using the same argument as in [31], we can obtain the following estimates (however, without any smallness assumption on $\xi$ ):

$$
\begin{aligned}
& \frac{d}{d t}\left(\left\|u_{1}-u_{2}\right\|_{L^{2}}^{2}+\lambda L\left\|\nabla\left(Q_{1}-Q_{2}\right)\right\|_{L^{2}}^{2}+\lambda\left\|Q_{1}-Q_{2}\right\|_{L^{2}}^{2}\right) \\
& \quad+\nu\left\|\nabla\left(u_{1}-u_{2}\right)\right\|_{L^{2}}^{2}+\lambda \Gamma L^{2}\left\|\Delta\left(Q_{1}-Q_{2}\right)\right\|_{L^{2}}^{2} \\
& \quad \leq h(t)\left(\left\|u_{1}-u_{2}\right\|_{L^{2}}^{2}+\lambda L\left\|\nabla\left(Q_{1}-Q_{2}\right)\right\|_{L^{2}}^{2}+\lambda\left\|Q_{1}-Q_{2}\right\|_{L^{2}}^{2}\right)
\end{aligned}
$$

where $h(t) \in L^{1}(0, T)$ is a certain time-integrable function. As a consequence, we have

$$
\begin{aligned}
& \left\|\left(u_{1}-u_{2}\right)(t)\right\|_{L^{2}}^{2}+\left\|\left(Q_{1}-Q_{2}\right)(t)\right\|_{H^{1}}^{2} \\
& \quad+\int_{0}^{t}\left(\left\|\nabla\left(u_{1}-u_{2}\right)(s)\right\|_{L^{2}}^{2}+\left\|\Delta\left(Q_{1}-Q_{2}\right)(s)\right\|_{L^{2}}^{2}\right) d s \\
& \quad \leq C e^{\int_{0}^{t} h(s) d s}\left(\left\|u_{01}-u_{02}\right\|_{L^{2}}^{2}+\left\|Q_{01}-Q_{02}\right\|_{H^{1}}^{2}\right) \quad \forall t \in(0, T),
\end{aligned}
$$

which implies that the global strong solution to the problem (1.1)-(1.5) is unique.

The proof of Theorem 6 is complete.

Remark 16. It seems impossible to prove any continuous dependence result on initial data for the strong solutions obtained above in the space $\mathbf{V} \times H^{2}$. Nevertheless, the estimate (4.21) implies that for any $\left(u_{0}, Q_{0}\right) \in \mathbf{V} \times H^{2}$, we are able to define a closed semigroup $\Sigma(t)$ for $t \geq 0$ (in the sense of [33]) by setting $(u(t), Q(t))=$ $\Sigma(t)\left(u_{0}, Q_{0}\right)$, where $(u, Q)$ is the global strong solution to problem (1.1)-(1.5).

5. Long-time behavior. In this section we investigate the long-time behavior of global strong solutions to problem (1.1)-(1.5) established in Theorem 6. The related study consists of two steps. First, we prove that for any global strong solution $(u(t), Q(t))$ its asymptotic limit as $t$ tends to infinity is unique. Then we provide an uniform estimate of the convergence rate. 
5.1. Characterization of $\boldsymbol{\omega}$-limit set. For any initial datum $\left(u_{0}, Q_{0}\right) \in \mathbf{V} \times$ $H^{2}\left(\mathbb{T}^{2}, S_{0}^{(2)}\right)$, we denote its $\omega$-limit set by

$$
\begin{aligned}
\omega\left(u_{0}, Q_{0}\right) \stackrel{\text { def }}{=}\left\{\left(u_{\infty}, Q_{\infty}\right) \mid \exists\left\{t_{n}\right\} \nearrow \infty: u\left(t_{n}\right) \rightarrow u_{\infty} \text { in } L^{2},\right. \\
\left.Q\left(t_{n}\right) \rightarrow Q_{\infty} \text { in } H^{1} \text { as } n \rightarrow \infty\right\} .
\end{aligned}
$$

On the other hand, we denote the set of steady states by

$$
\begin{aligned}
\mathfrak{S}=\left\{Q_{*}: L \Delta Q_{*}-a Q_{*}-\right. & c \operatorname{tr}\left(Q_{*}^{2}\right) Q_{*}=0, \quad Q_{*} \in S_{0}^{(2)} \\
& \text { and } \left.Q_{*}\left(x+e_{i}\right)=Q_{*}(x) \text { in } \mathbb{T}^{2}\right\} .
\end{aligned}
$$

Remark 17. Since the free energy $\mathcal{F}(Q)$ given by (1.6) is bounded from below, using the classical variational method and the elliptic regularity theorem, it is easy to see that the set $\mathfrak{S}$ is nonempty. Besides, every $Q_{*} \in \mathfrak{S}$ is a critical point of $\mathcal{F}(Q)$.

Next, by virtue of the properties of the $\omega$-limit set $\omega\left(u_{0}, Q_{0}\right)$ as well as the higherorder energy term $\mathcal{A}(t)$, we have the following.

Lemma 18. Suppose that the assumptions in Theorem 6 are satisfied. For any initial datum $\left(u_{0}, Q_{0}\right) \in \mathbf{V} \times H^{2}\left(\mathbb{T}^{2}, S_{0}^{(2)}\right)$, the $\omega$-limit set $\omega\left(u_{0}, Q_{0}\right)$ is a nonempty bounded subset in $\mathbf{V} \times H^{2}\left(\mathbb{T}^{2}, S_{0}^{(2)}\right)$ that satisfies

$$
\omega\left(u_{0}, Q_{0}\right) \in\left\{\left(0, Q_{*}\right): Q_{*} \in \mathfrak{S}\right\},
$$

and the total energy $\mathcal{E}(t)$ is a constant on $\omega\left(u_{0}, Q_{0}\right)$. Besides, the corresponding unique global strong solution $(u, Q)$ has the following decay property:

$$
\lim _{t \rightarrow+\infty}\left(\|u(t)\|_{H^{1}}+\|H(Q(t))\|_{L^{2}}\right)=0 .
$$

Proof. Since the global strong solution $(u, Q)$ obtained in Theorem 6 satisfies the higher-order energy inequality (3.6), using a similar argument as in section 4.2 , we get

$$
\mathcal{A}(t) \leq C \quad \forall t \geq 0,
$$

where $C>0$ depends on $\left\|u_{0}\right\|_{H^{1}},\left\|Q_{0}\right\|_{H^{2}}, L, \lambda, \nu, \Gamma, a, c, \xi$, and $\mathbb{T}^{2}$. As a consequence, it follows from (3.6) and (5.2) that

$$
\frac{d}{d t} \mathcal{A}(t) \leq C \quad \forall t \geq 0
$$

On the other hand, the energy identity $(2.7)$ for $(u, Q)$ yields that

$$
\int_{0}^{+\infty} \int_{\mathbb{T}^{2}} \nu|\nabla u|^{2}+\lambda \Gamma|H(Q)|^{2} d x d t \leq K_{0}
$$

where $K_{0}>0$ is a constant that only depends on $\left\|u_{0}\right\|_{L^{2}},\left\|Q_{0}\right\|_{H^{1}}, L, \lambda, a, c, \mathbb{T}^{2}$. This implies that $\int_{0}^{+\infty} \mathcal{A}(t) d t<+\infty$, which together with (5.3) leads to the decay property (5.1).

Since the total energy $\mathcal{E}(t)$ is nonincreasing in time and bounded from below by a generic constant, there exists a finite constant $\mathcal{F}_{\infty} \in \mathbb{R}$ such that

$$
\lim _{t \rightarrow+\infty} \mathcal{E}(t)=\mathcal{F}_{\infty}
$$

By the definition of $\omega\left(u_{0}, Q_{0}\right)$, it is easy to see that $\mathcal{E}(t)$ is equal to the constant $\mathcal{F}_{\infty}$ on the set $\omega\left(u_{0}, Q_{0}\right)$.

The proof is complete. 
5.2. Convergence to equilibrium. In general, we cannot directly conclude that each global strong solution of system (1.1)-(1.5) converges to a single equilibrium as $t \rightarrow+\infty$ because the set of steady states $\mathfrak{S}$ for $Q$-tensors can have a complicated structure. For instance, since we are working in the periodic torus $\mathbb{T}^{2}$, we may expect the dimension of the set $\mathfrak{S}$ to be at least 2 . However, we may establish a gradient inequality of Łojasiewicz-Simon type for the matrix valued function $Q$ and apply Simon's idea (see $[15,34]$ ) to achieve our goal.

To begin with, using (2.5) and (5.2), we have the uniform-in-time estimates

$$
\|u(t)\|_{H^{1}}+\|Q(t)\|_{H^{2}} \leq C \quad \forall t \geq 0
$$

Then, from Lemma 18 we infer that there exists an increasing unbounded sequence $\left\{t_{n}\right\}_{n \in N}$ and a matrix function $Q_{\infty} \in H^{2}\left(\mathbb{T}^{2}, S_{0}^{(2)}\right)$, such that

$$
\lim _{t_{n} \rightarrow+\infty}\left\|Q\left(t_{n}\right)-Q_{\infty}\right\|_{H^{1}}=0
$$

where $\left(0, Q_{\infty}\right) \in \omega\left(u_{0}, Q_{0}\right)$.

We now proceed to prove the convergence of $Q(t)$ to $Q_{\infty}$ for all time as $t \rightarrow+\infty$, which implies that the $\omega$-limit set $\omega\left(u_{0}, Q_{0}\right)$ is actually a singleton. For this purpose, the following Łojaciewicz-Simon type inequality plays an important role.

Lemma 19. Let $Q_{*} \in H^{1}\left(\mathbb{T}^{2}, S_{0}^{(2)}\right)$ be a critical point of the energy functional $\mathcal{F}(Q)$. Then there exist some constants $\theta \in\left(0, \frac{1}{2}\right)$ and $\beta>0$ depending on $Q_{*}$, such that for any $Q \in H^{1}\left(\mathbb{T}^{2}, S_{0}^{(2)}\right)$ satisfying $\left\|Q-Q_{*}\right\|_{H^{1}}<\beta$, we have

$$
\left\|L \Delta Q-a Q-c \operatorname{tr}\left(Q^{2}\right) Q\right\|_{\left(H^{1}\right)^{\prime}} \geq\left|\mathcal{F}(Q)-\mathcal{F}\left(Q_{*}\right)\right|^{1-\theta} .
$$

Here, $\left(H^{1}\left(\mathbb{T}^{2}, S_{0}^{(2)}\right)\right)^{\prime}$ is the dual space of $H^{1}\left(\mathbb{T}^{2}, S_{0}^{(2)}\right)$. form

Proof. We observe that any matrix function $Q \in S_{0}^{(2)}$ can be written into the

$$
Q(x)=\left(\begin{array}{cc}
p(x) & q(x) \\
q(x) & -p(x)
\end{array}\right)
$$

where $p, q$ are two scalar functions defined on $\mathbb{T}^{2}$. Now we introduce the vector $\tilde{Q}: \mathbb{T}^{2} \rightarrow \mathbb{R}^{2}$ defined by

$$
\tilde{Q}=\left(\begin{array}{c}
p \\
q
\end{array}\right)
$$

By direct computations, we can see that

$$
\begin{aligned}
\tilde{\mathcal{F}}(\tilde{Q}) & \stackrel{\text { def }}{=} \mathcal{F}(Q) \\
& =\int_{\mathbb{T}^{2}}\left[L\left(|\nabla p|^{2}+|\nabla q|^{2}\right)+a\left(p^{2}+q^{2}\right)+c\left(p^{2}+q^{2}\right)^{2}\right] d x .
\end{aligned}
$$

The corresponding Fréchet derivative of $\tilde{\mathcal{F}}$ with respect to $\tilde{Q}$ in $L^{2}$ is given by

$$
\frac{\delta \tilde{\mathcal{F}}}{\delta \tilde{Q}}=\left(\begin{array}{c}
\frac{\delta \tilde{\mathcal{F}}}{\delta p} \\
\frac{\delta \tilde{\mathcal{F}}}{\delta q}
\end{array}\right)=-2\left(\begin{array}{c}
L \Delta p-a p-2 c\left(p^{2}+q^{2}\right) p \\
L \Delta q-a q-2 c\left(p^{2}+q^{2}\right) q
\end{array}\right) .
$$

Copyright (c) by SIAM. Unauthorized reproduction of this article is prohibited. 
Let $\tilde{Q}_{*}=\left(\begin{array}{c}p_{*} \\ q_{*}\end{array}\right)$ be a critical point of $\mathcal{F}(\tilde{Q})$. Correspondingly, we can easily verify that $Q_{*}=\left(\begin{array}{cc}p_{*} & q_{*} \\ q_{*} & -p_{*}\end{array}\right)$ is a critical point of $\mathcal{F}(Q)$. Then, applying the Eojaciewisz-Simon inequality for vector valued functions derived in [21], we conclude that there exist some constants $\theta \in\left(0, \frac{1}{2}\right)$ and $\beta>0$ depending on $\tilde{Q}_{*}$ (and thus $Q_{*}$ ), such that the following inequality holds:

$$
\left\|\frac{\delta \tilde{\mathcal{F}}}{\delta \tilde{Q}}\right\|_{\left(H^{1}\right)^{\prime}} \geq\left|\tilde{\mathcal{F}}(\tilde{Q})-\tilde{\mathcal{F}}\left(\tilde{Q}_{*}\right)\right|^{1-\theta}
$$

for any $\tilde{Q} \in H^{1}\left(\mathbb{T}^{2}, \mathbb{R}^{2}\right)$, provided that $\left\|\tilde{Q}-\tilde{Q}_{*}\right\|_{H^{1}}<\frac{\beta}{2}$. Therefore, our conclusion (5.8) is an immediate consequence of the inequality (5.10).

The proof is complete.

Remark 20. Lemma 19 can be considered as an extended version for matrix valued functions of Simon's result in [34] for scalar functions. In the present case, there are two constraints (i.e., matrix symmetry and trace free) imposed on the matrix $Q \in S_{0}^{(2)}$, which might bring extra difficulties in the proof. However, due to the special structure of the $Q$-tensor in the 2D case (5.9), the possible difficulties can be avoided by reducing the problem to the vector case that has been treated in the literature.

Convergence of the order parameter $Q(t)$ can be proved by adapting the argument in [15] for parabolic equations, which relies on the following analysis lemma (see, e.g., [15, Lemma 7.1]).

Lemma 21. Let $\theta \in\left(0, \frac{1}{2}\right)$. Assume that $Z(t) \geq 0$ is a measurable function on $(0,+\infty), Z(t) \in L^{2}(0,+\infty)$ and there exist $C>0$ and $t_{0} \geq 0$ such that

$$
\int_{t}^{\infty} Z^{2}(s) d s \leq C Z(t)^{\frac{1}{1-\theta}} \text { for a.e. } t \geq t_{0} .
$$

Then $Z(t) \in L^{1}\left(t_{0},+\infty\right)$.

By Lemma 19, for each element $\left(0, Q_{\infty}\right) \in \omega\left(u_{0}, Q_{0}\right)$, there exist some constants $\beta_{Q_{\infty}}>0$ and $\theta_{Q_{\infty}} \in\left(0, \frac{1}{2}\right)$ such that the gradient inequality (5.8) holds for

$$
Q \in \mathbf{B}_{\beta_{Q_{\infty}}}\left(Q_{\infty}\right) \stackrel{\text { def }}{=}\left\{Q \in H^{1}\left(\mathbb{T}^{2}, S_{0}^{(2)}\right),\left\|Q-Q_{\infty}\right\|_{H^{1}}<\beta_{Q_{\infty}}\right\} .
$$

The union of balls $\{0\} \times\left\{\mathbf{B}_{\beta_{Q_{\infty}}}\left(Q_{\infty}\right):\left(0, Q_{\infty}\right) \in \omega\left(u_{0}, Q_{0}\right)\right\}$ forms an open cover of $\omega\left(u_{0}, Q_{0}\right)$. Due to the compactness of $\omega\left(u_{0}, Q_{0}\right)$ in $H^{1}$ (see Lemma 18), there exists a finite subcover $\{0\} \times\left\{\mathbf{B}_{\beta_{i}}\left(Q_{\infty}^{i}\right): i=1,2, \ldots, m\right\}$ of $\omega\left(u_{0}, Q_{0}\right)$ in $H^{1}$, where the constants $\beta_{i}, \theta_{i}$ corresponding to the limit point $Q_{\infty}^{i}$ (and thus a critical point of $\mathcal{F}(Q))$ in Lemma 19 are indexed by $i$. From the definition of $\omega\left(u_{0}, Q_{0}\right)$, there exists a sufficiently large $t_{0} \gg 1$ such that the global strong solution $Q(t)$ satisfies

$$
Q(t) \in \mathcal{U} \stackrel{\text { def }}{=} \bigcup_{i=1}^{m} \mathbf{B}_{\beta_{i}}\left(Q_{\infty}^{i}\right) \quad \text { for } t \geq t_{0} .
$$

Taking $\theta=\min _{i=1}^{m}\left\{\theta_{i}\right\} \in\left(0, \frac{1}{2}\right)$ and using Lemma 19 and convergence of the total energy $\mathcal{E}(t)$ (see (5.5)), we deduce, for all $t \geq t_{0}$,

$$
\begin{aligned}
\left|\mathcal{F}(Q(t))-\mathcal{F}_{\infty}\right|^{1-\theta} & \leq\left\|L \Delta Q(t)-a Q(t)-c \operatorname{tr}\left(Q(t)^{2}\right) Q(t)\right\|_{\left(H^{1}\right)^{\prime}} \\
& \leq\|H(Q(t))\|_{L^{2}} .
\end{aligned}
$$

Copyright (c) by SIAM. Unauthorized reproduction of this article is prohibited. 
Therefore, it holds that

$$
\begin{aligned}
\left(\mathcal{E}(t)-\mathcal{F}_{\infty}\right)^{1-\theta} & \leq\left(\frac{1}{2}\|u(t)\|_{L^{2}}^{2}+\left|\mathcal{F}(Q(t))-\mathcal{F}\left(Q_{\infty}\right)\right|\right)^{1-\theta} \\
& \leq\left(\frac{1}{2}\|u\|_{L^{2}}^{2}+\|H(Q(t))\|_{L^{2}}^{\frac{1}{1-\theta}}\right)^{1-\theta} \\
& \leq C\|u(t)\|_{L^{2}}^{2(1-\theta)}+C\|H(Q(t))\|_{L^{2}} \\
& \leq C\left(\|u(t)\|_{L^{2}}+\|H(Q(t))\|_{L^{2}}\right) \\
& \leq C \mathcal{A}^{\frac{1}{2}}(t) \quad \forall t \geq t_{0}
\end{aligned}
$$

in which we use the fact $0<\theta<\frac{1}{2}$ and the uniform estimate (5.6).

On the other hand, it follows from the energy inequality (2.4) that

$$
\mathcal{E}(t)-\mathcal{F}_{\infty} \geq \min \{\nu, \Gamma\} \int_{t}^{\infty} \mathcal{A}(s) d s \quad \forall t \geq t_{0} .
$$

As a consequence,

$$
\int_{t}^{\infty} \mathcal{A}(s) d s \leq C \mathcal{A}(t)^{\frac{1}{2(1-\theta)}} \quad \forall t \geq t_{0} .
$$

Taking $Z(t)=\mathcal{A}(t)^{\frac{1}{2}}$, from (5.17) and Lemma 21 we conclude that

$$
\int_{t_{0}}^{+\infty}\left(\|\nabla u(t)\|_{L^{2}}+\|H(Q(t))\|_{L^{2}}\right) d t \leq \int_{t_{0}}^{+\infty} \mathcal{A}(t)^{\frac{1}{2}} d t<+\infty .
$$

Then, by using (1.3) for $Q$, the uniform bounds on $\|u(t)\|_{H^{1}},\|Q(t)\|_{H^{2}}$, and the Sobolev embedding theorem $(d=2)$, we have

$$
\begin{aligned}
& \int_{t_{0}}^{\infty}\left\|Q_{t}(t)\right\|_{L^{2}} d t \\
& \quad \leq \int_{t_{0}}^{\infty}\left(\|u \cdot \nabla Q\|_{L^{2}}+\|S(\nabla u, Q)\|_{L^{2}}+\Gamma\|H(Q)\|_{L^{2}}\right) d t \\
& \quad \leq C \int_{t_{0}}^{\infty}\left[\|u\|_{L^{4}}\|\nabla Q\|_{L^{4}}+\|\nabla u\|_{L^{2}}\left(\|Q\|_{L^{\infty}}^{2}+1\right)+\|H(Q)\|_{L^{2}}\right] d \tau \\
& \quad \leq C \int_{t_{0}}^{\infty}\left(\|\nabla u(t)\|_{L^{2}}+\|H(Q)(t)\|_{L^{2}}\right) d t \\
& \quad<+\infty
\end{aligned}
$$

which indicates that $Q(t)$ converges in $L^{2}\left(\mathbb{T}^{2}\right)$ as $t \rightarrow+\infty$. Combining the sequential convergence result (5.7), it is easy to check that

$$
\lim _{t \rightarrow+\infty}\left\|Q(t)-Q_{\infty}\right\|_{L^{2}}=0
$$

Next, by the uniform bound on $\|Q(t)\|_{H^{2}}$ (see (5.6)) and (5.20), from the standard interpolation we obtain that

$$
\lim _{t \rightarrow+\infty}\left\|Q(t)-Q_{\infty}\right\|_{H^{1}}=0
$$

Copyright $@$ by SIAM. Unauthorized reproduction of this article is prohibited. 
Finally, observing the fact that

$$
\begin{aligned}
& \left\|\Delta Q-\Delta Q_{\infty}\right\|_{L^{2}} \\
& \quad \leq \frac{1}{L}\left\|H(Q)-H\left(Q_{\infty}\right)\right\|_{L^{2}}+\frac{1}{L}\left\|a Q+c \operatorname{tr}\left(Q^{2}\right) Q-a Q_{\infty}-c \operatorname{tr}\left(Q_{\infty}^{2}\right) Q_{\infty}\right\|_{L^{2}} \\
& \quad \leq \frac{1}{L}\|H(Q)\|_{L^{2}}+C\left\|Q-Q_{\infty}\right\|_{H^{1}},
\end{aligned}
$$

we further deduce from Lemma 18 and (5.21) that

$$
\lim _{t \rightarrow+\infty}\left\|Q(t)-Q_{\infty}\right\|_{H^{2}}=0 .
$$

5.3. Convergence rate. In what follows, we derive uniform estimates on the convergence rate. First, the rate on lower-order norm $\left\|Q(t)-Q_{\infty}\right\|_{L^{2}}$ follows from the Łojasiewicz-Simon approach (cf. [18]). To this end, we infer from the basic energy law (2.4), (5.5), and (5.15) that

$$
\frac{d}{d t}\left(\mathcal{E}(t)-\mathcal{F}_{\infty}\right)^{\theta}+C\left(\|\nabla u\|_{L^{2}}+\|H(Q)\|_{L^{2}}\right) \leq 0 \quad \forall t \geq t_{0}
$$

and also

$$
\frac{d}{d t}\left(\mathcal{E}(t)-\mathcal{F}_{\infty}\right)+C\left(\mathcal{E}(t)-\mathcal{F}_{\infty}\right)^{2(1-\theta)} \leq 0 \quad \forall t \geq t_{0} .
$$

As a consequence of (5.24), we can deduce the rate on energy decay:

$$
0 \leq \mathcal{E}(t)-\mathcal{F}_{\infty} \leq C(1+t)^{-\frac{1}{1-2 \theta}} \quad \forall t \geq t_{0} .
$$

Then similar to (5.19), on $(t,+\infty)$, where $t \geq t_{0}$, it follows from (5.23) that

$$
\begin{aligned}
\int_{t}^{\infty}\left\|Q_{t}(\tau)\right\|_{L^{2}} d \tau & \leq C \int_{t}^{\infty}\left(\|\nabla u(\tau)\|_{L^{2}}+\|H(Q)(\tau)\|_{L^{2}}\right) d \tau \\
& \leq\left(\mathcal{E}(t)-\mathcal{F}_{\infty}\right)^{\theta} \\
& \leq C(1+t)^{-\frac{\theta}{1-2 \theta}} \quad \forall t \geq t_{0},
\end{aligned}
$$

which further implies

$$
\left\|Q(t)-Q_{\infty}\right\|_{L^{2}} \leq C(1+t)^{-\frac{\theta}{1-2 \theta}} \quad \forall t \geq 0 .
$$

Higher-order estimates on the convergence rate can be achieved by constructing proper differential inequalities via the energy method (see, e.g., [41] for the simplified liquid crystal system). The key idea relies on the use of the basic energy law (2.4) combined with the higher-order energy inequality (3.6).

It follows from Lemma 18 that the limit system of the problem (1.1)-(1.5) takes the form

$$
\begin{aligned}
\nabla P_{\infty} & =-\lambda \nabla \cdot\left(\nabla Q_{\infty} \odot \nabla Q_{\infty}\right), \quad x \in \mathbb{T}^{2}, \\
H\left(Q_{\infty}\right) & =0, \quad x \in \mathbb{T}^{2}
\end{aligned}
$$

subject to periodic boundary conditions. Subtracting the stationary problem (5.26)(5.27) from the evolutionary problem (1.1)-(1.5) and then testing the velocity equation 
by $u$ and the $Q$-equation by $-\lambda\left(H(Q)-H\left(Q_{\infty}\right)\right)$, respectively, adding the results together and integrating over $\mathbb{T}^{2}$, we infer from (2.4) that

$$
\begin{aligned}
\frac{1}{2} \frac{d}{d t} & \left(\|u\|_{L^{2}}^{2}+\lambda L\left\|\nabla Q-\nabla Q_{\infty}\right\|_{L^{2}}^{2}\right) \\
& +\lambda \frac{d}{d t}\left(\int_{\mathbb{T}^{2}}\left[f_{B}(Q)-f_{B}\left(Q_{\infty}\right)-f_{B}^{\prime}\left(Q_{\infty}\right)\left(Q-Q_{\infty}\right)\right] d x\right) \\
& +\nu\|\nabla u\|_{L^{2}}^{2}+\lambda \Gamma\|H(Q)\|_{L^{2}}^{2} \\
= & -\lambda L \int_{\mathbb{T}^{2}} u_{i} \nabla_{j}\left[\nabla_{i}\left(Q_{\infty}\right)_{k l} \nabla_{j}\left(Q_{\infty}\right)_{k l}\right] d x \\
= & -\lambda \int_{\mathbb{T}^{2}} u_{i} \nabla_{i}\left(Q_{\infty}\right)_{k l}\left(L \Delta\left(Q_{\infty}\right)_{k l}-\left[f_{B}^{\prime}\left(Q_{\infty}\right)\right]_{k l}\right) d x \\
& -\lambda \int_{\mathbb{T}^{2}} u_{i} \nabla_{i}\left(Q_{\infty}\right)_{k l}\left[f_{B}^{\prime}\left(Q_{\infty}\right)\right]_{k l} d x-\frac{\lambda L}{2} \int_{\mathbb{T}^{2}} u_{i} \nabla_{i}\left|Q_{\infty}\right|^{2} d x \\
= & 0
\end{aligned}
$$

where $f_{B}^{\prime}(Q)=a Q+c Q \operatorname{tr}\left(Q^{2}\right)$. On the other hand, testing (1.3) by $Q-Q_{\infty}$, from the uniform estimate (5.6), the Hölder inequality, and the Sobolev embedding theorem $(d=2)$, we conclude that

$$
\begin{aligned}
\frac{1}{2} \frac{d}{d t}\left\|Q-Q_{\infty}\right\|_{L^{2}}^{2}+\Gamma L\left\|\nabla\left(Q-Q_{\infty}\right)\right\|_{L^{2}}^{2} \\
=\int_{\mathbb{T}^{2}}[-u \cdot \nabla Q+S(\nabla u, Q)]:\left(Q-Q_{\infty}\right) d x \\
\quad-\Gamma \int_{\mathbb{T}^{2}}\left(f_{B}^{\prime}(Q)-f_{B}^{\prime}\left(Q_{\infty}\right)\right):\left(Q-Q_{\infty}\right) d x \\
\leq C\|u\|_{L^{4}}\|\nabla Q\|_{L^{4}}\left\|Q-Q_{\infty}\right\|_{L^{2}}+C\|u\|_{L^{2}}\left(\|Q\|_{L^{\infty}}^{2}+1\right)\left\|Q-Q_{\infty}\right\|_{L^{2}} \\
\quad+C \int_{\mathbb{T}^{2}} \int_{0}^{1} f_{B}^{\prime \prime}\left(s Q+(1-s) Q_{\infty}\right)\left(Q-Q_{\infty}\right):\left(Q-Q_{\infty}\right) d s d x \\
\leq \epsilon_{1}\|\nabla u\|_{L^{2}}^{2}+C_{1}\left\|Q-Q_{\infty}\right\|_{L^{2}}^{2},
\end{aligned}
$$

where $\epsilon_{1}>0$ is a small constant to be determined below.

Multiplying (5.29) by a constant $\mu>0$ and adding the resultant to (5.28), we get

$$
\begin{aligned}
& \frac{d}{d t} \mathcal{Y}(t)+\left(\nu-\mu \epsilon_{1}\right)\|\nabla u\|_{L^{2}}^{2}+\lambda \Gamma\|H(Q)\|_{L^{2}}^{2}+\mu \Gamma L\left\|\nabla\left(Q-Q_{\infty}\right)\right\|_{L^{2}}^{2} \\
& \quad \leq C_{1} \mu\left\|Q-Q_{\infty}\right\|_{L^{2}}^{2},
\end{aligned}
$$

where

$$
\begin{aligned}
\mathcal{Y}(t) \stackrel{\text { def }}{=} & \frac{1}{2}\|u(t)\|_{L^{2}}^{2}+\frac{\lambda L}{2}\left\|\nabla Q(t)-\nabla Q_{\infty}\right\|_{L^{2}}^{2}+\frac{\mu}{2}\left\|Q(t)-Q_{\infty}\right\|_{L^{2}}^{2} \\
& +\lambda \int_{\mathbb{T}^{2}}\left[f_{B}(Q(t))-f_{B}\left(Q_{\infty}\right)-f_{B}^{\prime}\left(Q_{\infty}\right)\left(Q(t)-Q_{\infty}\right)\right] d x
\end{aligned}
$$

Copyright $@$ by SIAM. Unauthorized reproduction of this article is prohibited. 
It follows from the Newton-Leibinz formula and (5.6) that

$$
\begin{aligned}
& \left|\int_{\mathbb{T}^{2}}\left[f_{B}(Q)-f_{B}\left(Q_{\infty}\right)-f_{B}^{\prime}\left(Q_{\infty}\right):\left(Q-Q_{\infty}\right)\right] d x\right| \\
& \quad \leq \mid \int_{\mathbb{T}^{2}} \int_{0}^{1} s \int_{0}^{1} f_{B}^{\prime \prime}\left(\rho\left(s Q+(1-s) Q_{\infty}\right)+(1-\rho) Q_{\infty}\right)\left(Q-Q_{\infty}\right) \\
& \quad \leq\left\|f_{B}^{\prime \prime}\right\|_{L^{\infty}}\left\|Q-Q_{\infty}\right\|_{L^{2}}^{2} \\
& \left.\quad \leq C_{2} \| Q-Q_{\infty}\right) d \rho d s d x \mid \\
& \quad L_{L^{2}} .
\end{aligned}
$$

Thus we shall choose $\mu \geq 2+2 \lambda C_{2}>0$ so that there exist constants $k_{1}>k_{2}>0$,

$$
k_{1}\left(\|u(t)\|_{L^{2}}^{2}+\left\|Q(t)-Q_{\infty}\right\|_{H^{1}}^{2}\right) \geq \mathcal{Y}(t) \geq k_{2}\left(\|u(t)\|_{L^{2}}^{2}+\left\|Q(t)-Q_{\infty}\right\|_{H^{1}}^{2}\right) .
$$

Once $\mu$ is fixed, we take $\epsilon_{1}=\frac{\nu}{2 \mu}$ in the inequality (5.30). Then it follows from (5.33) that there exist some constants $C_{3}, C_{4}>0$ such that

$$
\frac{d}{d t} \mathcal{Y}(t)+C_{3}[\mathcal{Y}(t)+\mathcal{A}(t)] \leq C_{4}\left\|Q(t)-Q_{\infty}\right\|_{L^{2}}^{2},
$$

which together with (5.25) yields

$$
\mathcal{Y}(t) \leq C(1+t)^{-\frac{2 \theta}{1-2 \theta}} \quad \forall t \geq 0 .
$$

In view of (5.33), we can deduce that

$$
\|u(t)\|_{L^{2}}+\left\|Q(t)-Q_{\infty}\right\|_{H^{1}} \leq C(1+t)^{-\frac{\theta}{1-2 \theta}} \quad \forall t \geq 0 .
$$

Last, it follows from the higher-order energy inequality (3.6) and the uniform estimate (5.6) that

$$
\frac{d}{d t} \mathcal{A}(t) \leq C_{5} \mathcal{A}(t)
$$

Multiplying (5.37) with $\alpha=\frac{C_{3}}{2 C_{5}}$ and adding the resultant with (5.34), we deduce

$$
\frac{d}{d t}[\mathcal{Y}(t)+\alpha \mathcal{A}(t)]+C_{6}[\mathcal{Y}(t)+\alpha \mathcal{A}(t)] \leq C(1+t)^{-\frac{2 \theta}{1-2 \theta}} .
$$

As a consequence,

$$
\mathcal{Y}(t)+\alpha \mathcal{A}(t) \leq C(1+t)^{-\frac{2 \theta}{1-2 \theta}} \quad \forall t \geq 0,
$$

which together with the fact $\mathcal{Y}(t) \geq 0$ (see (5.33)) yields

$$
\mathcal{A}(t) \leq C(1+t)^{-\frac{2 \theta}{1-2 \theta}} \quad \forall t \geq 0 .
$$

Then from the definition of $\mathcal{A}(t)$ and estimates (5.36), (5.40), we can see that

$$
\|\nabla u(t)\|_{L^{2}}+\left\|\Delta Q(t)-\Delta Q_{\infty}\right\|_{L^{2}} \leq C(1+t)^{-\frac{\theta}{1-2 \theta}} \quad \forall t \geq 0 .
$$

Collecting the estimates (5.36) and (5.41), we arrive at our conclusion (2.17). The proof of Theorem 7 is complete.

Copyright $@$ by SIAM. Unauthorized reproduction of this article is prohibited. 
6. Appendix. In the appendix, we compute the time derivative of $\mathcal{A}(t)$. The subsequent calculations hold for both $2 \mathrm{D}$ and $3 \mathrm{D}$ cases.

Lemma 22. Suppose $d=2,3$. Let $(u, Q)$ be a smooth solution to the problem (1.1)-(1.5). Define

$$
\mathcal{A}(t)=\|\nabla u(t)\|_{L^{2}}^{2}+\lambda\|H(Q(t))\|_{L^{2}}^{2} .
$$

Then we have the following equality:

$$
\begin{aligned}
& \frac{1}{2} \frac{d}{d t} \mathcal{A}(t)+\nu\|\Delta u\|_{L^{2}}^{2}+\lambda \Gamma\|\nabla H(Q)\|_{L^{2}}^{2} \\
&= \int_{\mathbb{T}^{d}}(u \cdot \nabla u) \cdot \Delta u d x-2 \lambda \int_{\mathbb{T}^{d}} \nabla_{l} u_{k} \nabla_{l} \nabla_{k} Q_{i j} H_{i j} d x \\
&+\frac{\lambda}{L} \int_{\mathbb{T}^{d}} u_{k} \nabla_{k} F_{i j} H_{i j} d x \\
&-2 \lambda \int_{\mathbb{T}^{d}} \nabla_{j} u_{i}\left(\nabla_{l} Q_{k j} \nabla_{l} H_{i k}-\nabla_{l} Q_{i k} \nabla_{l} H_{k j}\right) d x \\
&-\lambda \int_{\mathbb{T}^{d}} \nabla_{j} u_{i}\left(\Delta Q_{k j} H_{i k}-\Delta Q_{i k} H_{k j}\right) d x \\
&+\lambda \xi \int_{\mathbb{T}^{d}}(D \Delta Q+\Delta Q D): H(Q) d x+4 \lambda \xi \int_{\mathbb{T}^{d}} \nabla_{l} D_{i k} \nabla_{l} Q_{k j} H_{i j} d x \\
&-2 \lambda \xi \int_{\mathbb{T}^{d}} \Delta\left(Q_{k l} Q_{j i}\right) \nabla_{j} u_{i} H_{k l} d x \\
&-4 \lambda \xi \int_{\mathbb{T}^{d}} \nabla_{m}\left(Q_{k l} Q_{j i}\right) \nabla_{m} \nabla_{j} u_{i} H_{k l} d x \\
&-\lambda \int_{\mathbb{T}^{d}} \frac{\partial F(Q)}{\partial Q}(u \cdot \nabla Q): H(Q) d x+\lambda \int_{\mathbb{T}^{d}} \frac{\partial F(Q)}{\partial Q} S(\nabla u, Q): H(Q) d x \\
&+\lambda \Gamma \int_{\mathbb{T}^{d}} \frac{\partial F(Q)}{\partial Q} H(Q): H(Q) d x \\
& 12 \\
& \stackrel{\text { def }}{=} \sum_{i=1} J_{i},
\end{aligned}
$$

where $F(Q)$ is given by

$$
\begin{aligned}
F(Q) & =-a Q+b\left(Q^{2}-\frac{1}{d} \operatorname{tr}\left(Q^{2}\right) \mathbb{I}\right)-c Q \operatorname{tr}\left(Q^{2}\right) \\
& =-\frac{\partial f_{B}(Q)}{\partial Q}-\frac{b}{d} \operatorname{tr}\left(Q^{2}\right) \mathbb{I} .
\end{aligned}
$$

Proof. Using (1.1), (1.3), and integration by parts, we have

$$
\begin{aligned}
& \frac{1}{2} \frac{d}{d t}\|\nabla u\|_{L^{2}}^{2}+\nu\|\Delta u\|_{L^{2}}^{2} \\
& \quad=\int_{\mathbb{T}^{d}}(u \cdot \nabla u) \cdot \Delta u d x-\lambda \int_{\mathbb{T}^{d}}(\nabla \cdot \sigma) \cdot \Delta u d x-\lambda \int_{\mathbb{T}^{d}}(\nabla \cdot \tau) \cdot \Delta u d x \\
& \quad \stackrel{\text { def }}{=} I_{1}+I_{2}+I_{3},
\end{aligned}
$$

where

$$
I_{2}=-\lambda \int_{\mathbb{T}^{d}} \nabla_{j}\left(Q_{i k} H_{k j}-H_{i k} Q_{k j}\right) \Delta u_{i} d x
$$

Copyright $@$ by SIAM. Unauthorized reproduction of this article is prohibited. 
and

$$
\begin{aligned}
I_{3}= & \lambda L \int_{\mathbb{T}^{d}} \nabla_{j}\left(\nabla_{i} Q_{k l} \nabla_{j} Q_{k l}\right) \Delta u_{i} d x \\
& +\lambda \xi \int_{\mathbb{T}^{d}} \nabla_{j}\left(Q_{i k} H_{k j}+H_{i k} Q_{k j}+\frac{2}{d} H_{i j}\right) \Delta u_{i} d x \\
& -2 \lambda \xi \int_{\mathbb{T}^{d}} \nabla_{j}\left(Q_{k l} H_{k l} Q_{i j}+\frac{1}{d} Q_{k l} H_{k l} \delta_{i j}\right) \Delta u_{i} d x \\
& \stackrel{\text { def }}{=} I_{3 a}+I_{3 b}+I_{3 c} .
\end{aligned}
$$

As in [2, A.3], using the incompressibility condition $\nabla \cdot u=0$, the definition of $F(Q)$ (see (6.3)), and the fact $\nabla \operatorname{tr}(Q)=0$, we get

$$
\begin{aligned}
I_{3 a}= & \lambda \int_{\mathbb{T}^{d}} \nabla_{i} Q_{k l}\left(H_{k l}-F_{k l}\right) \Delta u_{i} d x+\lambda L \int_{\mathbb{T}^{d}} \nabla_{j} \nabla_{i} Q_{k l} \nabla_{j} Q_{k l} \Delta u_{i} d x \\
= & \lambda \int_{\mathbb{T}^{d}} \nabla_{i} Q_{k l} H_{k l} \Delta u_{i} d x+\lambda \int_{\mathbb{T}^{d}} \nabla f_{B}(Q) \cdot \Delta u d x \\
& +\frac{\lambda b}{d} \int_{\mathbb{T}^{d}} \operatorname{tr}\left(Q^{2}\right) \nabla \operatorname{tr}(Q) \cdot \Delta u d x+\frac{\lambda L}{2} \int_{\mathbb{T}^{d}}\left(\nabla|\nabla Q|^{2}\right) \cdot \Delta u d x \\
= & \lambda \int_{\mathbb{T}^{d}} \nabla_{i} Q_{k l} H_{k l} \Delta u_{i} d x .
\end{aligned}
$$

Using the symmetry of $Q$ and $H(Q)$ as well as the basic algebra for arbitrary matrices $A, B, C \in \mathbb{R}^{d \times d}$

$$
(A B): C=B:\left(A^{T} C\right)=A:\left(C B^{T}\right),
$$

we have

$$
\begin{aligned}
I_{3 b}= & -\frac{2 \lambda \xi}{d} \int_{\mathbb{T}^{d}} H_{i j} \nabla_{j} \Delta u_{i} d x-\lambda \xi \int_{\mathbb{T}^{d}}\left(Q_{i k} H_{k j}+H_{i k} Q_{k j}\right) \nabla_{j} \Delta u_{i} d x \\
= & -\frac{2 \lambda \xi}{d} \int_{\mathbb{T}^{d}} H_{i j} \Delta D_{i j} d x-\lambda \xi \int_{\mathbb{T}^{d}} Q_{i k} H_{k j} \Delta D_{i j} d x \\
& -\lambda \xi \int_{\mathbb{T}^{d}} H_{i k} Q_{k j} \Delta D_{i j} d x \\
= & -\lambda \xi \int_{\mathbb{T}^{d}}\left(\Delta D Q+Q \Delta D+\frac{2}{d} \Delta D\right): H(Q) d x .
\end{aligned}
$$

By the incompressibility condition $\nabla \cdot u=0$, it holds that

$$
\begin{aligned}
I_{3 c} & =-2 \lambda \xi \int_{\mathbb{T}^{d}} \nabla_{j}\left(Q_{k l} H_{k l} Q_{i j}\right) \Delta u_{i} d x-\frac{2 \lambda \xi}{d} \int_{\mathbb{T}^{d}} \nabla_{i}\left(Q_{k l} H_{k l}\right) \Delta u_{i} d x \\
& =-2 \lambda \xi \int_{\mathbb{T}^{d}} \nabla_{j}\left(Q_{k l} H_{k l} Q_{i j}\right) \Delta u_{i} d x .
\end{aligned}
$$

Copyright $\odot$ by SIAM. Unauthorized reproduction of this article is prohibited. 
On the other hand, it holds that

$$
\begin{aligned}
\frac{\lambda}{2} \frac{d}{d t}\|H(Q)\|_{L^{2}}^{2}+\lambda \Gamma\|\nabla H(Q)\|_{L^{2}}^{2} \\
=\lambda L \int_{\mathbb{T}^{d}} \Delta Q_{t}: H(Q) d x+\lambda \int_{\mathbb{T}^{d}} \partial_{t} F(Q): H(Q) d x+\lambda \Gamma\|\nabla H(Q)\|_{L^{2}}^{2} \\
=\lambda L \int_{\mathbb{T}^{d}} Q_{t}: \Delta H(Q) d x+\lambda \int_{\mathbb{T}^{d}} \partial_{t} F(Q): H(Q) d x+\lambda \Gamma\|\nabla H(Q)\|_{L^{2}}^{2} \\
=-\lambda \int_{\mathbb{T}^{d}} u_{k} \nabla_{k} Q_{i j} \Delta H_{i j} d x+\lambda \int_{\mathbb{T}^{d}} S(\nabla u, Q): \Delta H d x \\
\quad+\lambda \int_{\mathbb{T}^{d}} \partial_{t} F(Q): H(Q) d x \\
\quad \stackrel{\text { def }}{=} I_{4}+I_{5}+I_{6} .
\end{aligned}
$$

Then by the incompressibility condition $\nabla \cdot u=0$ and (1.3) we have

$$
\begin{aligned}
I_{4}= & -\lambda \int_{\mathbb{T}^{d}} u_{k} \nabla_{k} Q_{i j} \Delta H_{i j} d x \\
= & \lambda \int_{\mathbb{T}^{d}}\left(\nabla_{l} u_{k} \nabla_{k} Q_{i j}+u_{k} \nabla_{l} \nabla_{k} Q_{i j}\right) \nabla_{l} H_{i j} d x \\
= & -\lambda \int_{T^{d}} \Delta u_{k} \nabla_{k} Q_{i j} H_{i j} d x-2 \lambda \int_{\mathbb{T}^{d}} \nabla_{l} u_{k} \nabla_{l} \nabla_{k} Q_{i j} H_{i j} d x \\
& -\frac{\lambda}{L} \int_{\mathbb{T}^{d}} u_{k} \nabla_{k}\left(H_{i j}-F_{i j}\right) H_{i j} d x \\
= & -\lambda \int_{\mathbb{T}^{d}} \Delta u_{k} \nabla_{k} Q_{i j} H_{i j} d x-2 \lambda \int_{\mathbb{T}^{d}} \nabla_{l} u_{k} \nabla_{l} \nabla_{k} Q_{i j} H_{i j} d x \\
& +\frac{\lambda}{L} \int_{\mathbb{T}^{d}} u_{k} \nabla_{k} F_{i j} H_{i j} d x \\
\stackrel{\text { def }}{=} & I_{4 a}+I_{4 b}+I_{4 c}
\end{aligned}
$$

and

$$
\begin{aligned}
I_{5}= & \lambda \int_{\mathbb{T}^{d}}\left[(\xi D+\Omega)\left(Q+\frac{1}{d} \mathbb{I}\right)+\left(Q+\frac{1}{d} \mathbb{I}\right)(\xi D-\Omega)\right]: \Delta H d x \\
& -2 \lambda \xi \int_{\mathbb{T}^{d}} \operatorname{tr}(Q \nabla u)\left(Q+\frac{1}{d} \mathbb{I}\right): \Delta H d x \\
= & \lambda \int_{\mathbb{T}^{d}}(\Omega Q-Q \Omega): \Delta H d x+\lambda \xi \int_{\mathbb{T}^{d}}\left(D Q+Q D+\frac{2}{d} D\right): \Delta H d x \\
& -2 \lambda \xi \int_{\mathbb{T}^{d}} \operatorname{tr}(Q \nabla u)\left(Q+\frac{1}{d} \mathbb{I}\right): \Delta H d x \\
\stackrel{\text { def }}{=} & I_{5 a}+I_{5 b}+I_{5 c} .
\end{aligned}
$$

Using the symmetry properties of $Q, H$, and (6.8), after integration by parts, it is 
easy to check that

$$
\begin{aligned}
I_{5 a}= & \lambda \int_{\mathbb{T}^{d}}(\Omega Q-Q \Omega): \Delta H d x \\
= & \frac{\lambda}{2} \int_{\mathbb{T}^{d}}\left(\nabla u Q-\nabla^{T} u Q-Q \nabla u+Q \nabla^{T} u\right): \Delta H d x \\
= & \lambda \int_{\mathbb{T}^{d}} \nabla u:(\Delta H Q-Q \Delta H) d x \\
= & \lambda \int_{\mathbb{T}^{d}} \Delta\left(\nabla_{j} u_{i} Q_{k j}\right) H_{i k} d x-\lambda \int_{\mathbb{T}^{d}} \Delta\left(\nabla_{j} u_{i} Q_{i k}\right) H_{k j} d x \\
= & \lambda \int_{\mathbb{T}^{d}} \Delta \nabla_{j} u_{i}\left(Q_{k j} H_{i k}-Q_{i k} H_{k j}\right) d x \\
& +2 \lambda \int_{\mathbb{T}^{d}} \nabla_{l} \nabla_{j} u_{i}\left(\nabla_{l} Q_{k j} H_{i k}-\nabla_{l} Q_{i k} H_{k j}\right) d x \\
& +\lambda \int_{\mathbb{T}^{d}} \nabla_{j} u_{i}\left(\Delta Q_{k j} H_{i k}-\Delta Q_{i k} H_{k j}\right) d x \\
= & \lambda \int_{\mathbb{T}^{d}} \nabla_{j}\left(Q_{i k} H_{k j}-Q_{k j} H_{i k}\right) \Delta u_{i} d x \\
& -2 \lambda \int_{\mathbb{T}^{d}} \nabla_{j} u_{i}\left(\nabla_{l} Q_{k j} \nabla_{l} H_{i k}-\nabla_{l} Q_{i k} \nabla_{l} H_{k j}\right) d x \\
& -\lambda \int_{\mathbb{T}^{d}} \nabla_{j} u_{i}\left(\Delta Q_{k j} H_{i k}-\Delta Q_{i k} H_{k j}\right) d x .
\end{aligned}
$$

Moreover, using integration by parts again, we have

$$
\begin{aligned}
I_{5 b}= & \lambda \xi \int_{\mathbb{T}^{d}} \Delta\left(D Q+Q D+\frac{2}{d} D\right): H d x \\
= & \lambda \xi \int_{\mathbb{T}^{d}}\left(\Delta D Q+Q \Delta D+\frac{2}{d} \Delta D\right): H d x \\
& +\lambda \xi \int_{\mathbb{T}^{d}}(D \Delta Q+\Delta Q D): H d x+4 \lambda \xi \int_{\mathbb{T}^{d}} \nabla_{l} D_{i k} \nabla_{l} Q_{k j} H_{i j} d x .
\end{aligned}
$$

Next, for $I_{5 c}$, using the property $\operatorname{tr}(H(Q))=0$ and after integration by parts, we obtain that

$$
\begin{aligned}
I_{5 c}= & -2 \lambda \xi \int_{\mathbb{T}^{d}} \Delta[\operatorname{tr}(Q \nabla u) Q]: H d x \\
= & -2 \lambda \xi \int_{\mathbb{T}^{d}} \Delta\left(Q_{k l} Q_{j i} \nabla_{j} u_{i}\right) H_{k l} d x \\
= & -2 \lambda \xi \int_{\mathbb{T}^{d}} Q_{k l} Q_{j i} \nabla_{j} \Delta u_{i} H_{k l} d x-2 \lambda \xi \int_{\mathbb{T}^{d}} \Delta\left(Q_{k l} Q_{j i}\right) \nabla_{j} u_{i} H_{k l} d x \\
& -4 \lambda \xi \int_{\mathbb{T}^{d}} \nabla_{m}\left(Q_{k l} Q_{j i}\right) \nabla_{m} \nabla_{j} u_{i} H_{k l} d x \\
= & 2 \lambda \xi \int_{\mathbb{T}^{d}} \nabla_{j}\left(Q_{k l} H_{k l} Q_{j i}\right) \Delta u_{i} d x-2 \lambda \xi \int_{\mathbb{T}^{d}} \Delta\left(Q_{k l} Q_{j i}\right) \nabla_{j} u_{i} H_{k l} d x \\
& -4 \lambda \xi \int_{\mathbb{T}^{d}} \nabla_{m}\left(Q_{k l} Q_{j i}\right) \nabla_{m} \nabla_{j} u_{i} H_{k l} d x .
\end{aligned}
$$

Copyright $\odot$ by SIAM. Unauthorized reproduction of this article is prohibited. 
Finally, using (1.3), the term $I_{6}$ can be expressed as

$$
\begin{aligned}
I_{6} & =\lambda \int_{\mathbb{T}^{d}} \frac{\partial F(Q)}{\partial Q} \partial_{t} Q: H(Q) d x \\
& =\lambda \int_{\mathbb{T}^{d}} \frac{\partial F(Q)}{\partial Q}(-u \cdot \nabla Q+S(\nabla u, Q)+\Gamma H(Q)): H(Q) d x .
\end{aligned}
$$

In summary, we find the following special cancellations in those highly nonlinear terms:

(a) The term $I_{2}$ (i.e., (6.5)) cancels with the first term in (6.13).

(b) The term $I_{3 a}$ (i.e., (6.7)) cancels with the term $I_{4 a}$ in (6.12).

(c) The term $I_{3 b}$ (i.e., (6.9)) cancels with the first term in (6.14).

(d) The term $I_{3 c}$ (i.e., (6.10)) cancels with the first term in (6.15).

Taking into account the above cancellation relations, we can easily conclude (6.2).

The proof is complete.

Remark 23. We note that in the above cancellations, the relation (a) is the same as for the simpler case with $\xi=0$ (see, e.g., [2]). However, for the general case $\xi \neq 0$, we have found extra relations (b)-(d) between higher-order nonlinear terms of the full Navier-Stokes and Q-tensor system (1.1)-(1.5).

Acknowledgment. We would like to acknowledge the helpful comments from anonymous reviewers.

\section{REFERENCES}

[1] H. Abels, G. Dolzmann, And Y.-N. Liu, Well-posedness of a fully coupled Navier-Stokes/Qtensor system with inhomogeneous boundary data, SIAM J. Math. Anal., 46 (2014), pp. 3050-3077.

[2] H. Abels, G. Dolzmann, And Y.-N. Liu, Strong solutions for the Beris-Edwards model for nematic liquid crystals with homogeneous Dirichlet boundary conditions, Adv. Differential Equations., 21 (2015), pp. 109-153.

[3] J. Ball And A. Majumdar, Nematic liquid crystals: From Maier-Saupe to a continuum theory, Mol. Cryst. Liq. Cryst., 525 (2010), pp. 1-11.

[4] A.-N. Beris AND B.-J. EdWARDS, Thermodynamics of Flowing Systems with Internal Microstructure, Ox. Eng. Sci. Ser. 36, Oxford University Press, Oxford, 1994.

[5] H. BrÉzIS ANd T. Gallouet, Nonlinear Schrödinger Evolution Equations, Nonlinear Anal., 4 (1980), pp. 677-681.

[6] C. Cavaterra And E. Rocca, On a $3 D$ isothermal model for nematic liquid crystals accounting for stretching terms, Z. Angew. Math. Phys., 64 (2013), pp. 69-82.

[7] C. Cavaterra, E. Rocca, And H. Wu, Global weak solution and blow-up criterion of the general Ericksen-Leslie system for nematic liquid crystal flows, J. Differential Equations., 255 (2013), pp. 24-57.

[8] Y. Chemin And C.-J. Xu, Inclusions de Sobolev en calcul de Weyl-Hörmander et champs de vecteurs sous-elliptiques, Ann. Sci. Éc. Norm. Supér (4), 30 (1997), pp. 719-751.

[9] X. F. Chen AND X. XU, Existence and uniqueness of global classical solutions of a gradient flow of the Landau-de Gennes energy, Proc. Amer. Math. Soc., 144 (2016), pp. 1251-1263.

[10] M. M. Dai, E. Feireisl, E. Rocca, G. Schimperna, and M. Schonbek, On asymptotic isotropy for a hydrodynamic model of liquid crystals, preprint, arXiv:1409.7499, 2014.

[11] F. De Anna And A. Zarnescu, Uniqueness of Weak Solutions of the Full Coupled NavierStokes and Q-Tensor System in 2D, preprint, arXiv:1509.0059, 2015.

[12] P. G. De Gennes and J. Prost, The Physics of Liquid Crystals, Oxford University Press, Oxford, 1993.

[13] E. Feireisl, E. Rocca, G. Schimperna, and A. Zarnescu, Evolution of non-isothermal Landau-de Gennes nematic liquid crystals flows with singular potential, Commun. Math. Sci., 12 (2014), pp. 317-343.

Copyright (c) by SIAM. Unauthorized reproduction of this article is prohibited. 
[14] E. Feireisl, E. Rocca, G. Schimperna, And A. Zarnescu, Nonisothermal nematic liquid crystal flows with the Ball-Majumdar free energy, Ann. Mat. Pura Appl., 194 (2015), pp. 1269-1299.

[15] E. Feireisl and F. Simondon, Convergence for semilinear degenerate parabolic equations in several space dimensions, J. Dynam. Differential Equations, 12 (2000), pp. 647-673.

[16] F. Guillén-GonzÁlez And M. A. RodríGuez-Bellido, Weak time regularity and uniqueness for a Q-tensor model, SIAM J. Math. Anal., 46 (2014), pp. 3540-3567.

[17] F. Guillén-GonzÁlez And M. A. RodríGuez-Bellido, Weak solutions for an initial-boundary Q-tensor problem related to liquid crystals, Nonlinear Anal., 112 (2015), pp. 84-104.

[18] A. Haraux And M. A. Jendoubi, Decay estimates to equilibrium for some evolution equations with an analytic nonlinearity, Asymptot. Anal., 26 (2001), pp. 21-36.

[19] J.-R. Huang And S.-J. Ding, Global well-posedness for the dynamical Q-tensor model of liquid crystals, Sci. China Math., 58 (2015), pp. 1349-1366.

[20] J.-R. Huang, F.-H. Lin, AND C.-Y. WANG, Regularity and existence of global solutions to the Ericksen-Leslie system in $\mathbb{R}^{2}$, Comm. Math. Phys., 331 (2014), pp. 805-850.

[21] S.-Z. Huang, Gradient Inequalities, with Applications to Asymptotic Behavior and Stability of Gradient-Like Systems, Math. Surveys Monogr. 126, AMS, Providence, RI, 2006.

[22] G. IYer, X. Xu, AND A. ZARnescu, Dynamic cubic instability in a $2 D$ Q-tensor model for liquid crystals, Math. Models Methods Appl. Sci., 25 (2015), pp. 1477-1517.

[23] O. A. Ladyzhenskaya, N. A. Solonnikov, and N. N. Uraltseva, Linear and quasilinear equations of parabolic type, Transl. Math. Monogr. 23, AMS, Providence, RI, 1968.

[24] F. LESLIE, Some constitutive equations for liquid crystals, Arch. Ration. Mech. Anal., 28 (1968), pp. $265-283$.

[25] F.-H. Lin And C. LiU, Nonparabolic dissipative system modeling the flow of liquid crystals, Comm. Pure Appl. Math., XLVIII (1995), pp. 501-537.

[26] F.-H. Lin AND C. LiU, Existence of solutions for the Ericksen-Leslie system, Arch. Ration. Mech. Anal., 154 (2000), pp. 135-156.

[27] A. Majumdar, Equilibrium order parameters of nematic liquid crystals in the Landau-de Gennes theory, European J. Appl. Math., 21 (2010), pp. 181-203.

[28] A. Majumdar and A. Zarnescu, Landau-de Gennes theory of nematic liquid crystals: The Oseen-Frank limit and beyond, Arch. Ration. Mech. Anal., 196 (2010), pp. 227-280.

[29] J. NĔCAS, Les méthodes Directes en Théorie des Équations Elliptiques, Academia, Prague, 1967.

[30] M. PAICU, Équation periodique de Navier-Stokes sans viscosité dans une direction, Comm. Partial Differential Equations, 30 (2005), pp. 1107-1140.

[31] M. Paicu And A. Zarnescu, Global existence and regularity for the full coupled Navier-Stokes and Q-tensor system, SIAM J. Math. Anal., 43 (2011), pp. 2009-2049.

[32] M. Paicu And A. Zarnescu, Energy dissipation and regularity for a coupled Navier-Stokes and Q-tensor system, Arch. Ration. Mech. Anal., 203 (2012), pp. 45-67.

[33] V. Pata and S. Zelik, A result on the existence of global attractors for semigroups of closed operators, Commun. Pure Appl. Anal., 6 (2007), pp. 481-486.

[34] L. Simon, Asymptotics for a class of nonlinear evolution equation with applications to geometric problems, Ann. of Math., 118 (1983), pp. 525-571.

[35] J. Simon, Compact sets in the space $L^{p}(0, T ; B)$, Ann. Mat. Pura Appl., 146 (1987), pp. 65-96.

[36] R. Temam, Navier-Stokes Equations and Nonlinear Functional Analysis, SIAM, Philadelphia, 1983.

[37] R. Temam, Infinite Dimensional Dynamical Systems in Mechanics and Physics, Springer, New York, 1997.

[38] W. Wang, P.-W. Zhang, and Z.-F. Zhang, Well-posedness of the Ericksen-Leslie system, Arch. Ration. Mech. Anal., 210 (2013), pp. 837-855.

[39] W. Wang, P.-W. Zhang, and Z.-F. Zhang, Rigorous derivation from Landau-de Gennes theory to Ericksen-Leslie theory, SIAM J. Math. Anal., 47 (2015), pp. 127-158.

[40] M. Wilkinson, Strict physicality of global weak solutions of a Navier-Stokes Q-tensor system with singular potential, Arch. Ration. Mech. Anal., 218 (2015), pp. 487-526.

[41] H. WU, Long-time behavior for nonlinear hydrodynamic system modeling the nematic liquid crystal flows, Discrete Contin. Dyn. Syst., 26 (2010), pp. 379-396.

[42] H. Wu, X. Xu, ANd C. Liu, On the general Ericksen-Leslie system: Parodi's relation, wellposedness and stability, Arch. Ration. Mech. Anal., 208 (2013), pp. 59-107.

Copyright $@$ by SIAM. Unauthorized reproduction of this article is prohibited. 